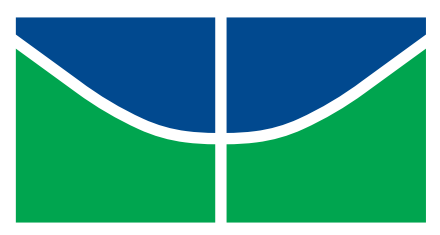

\author{
Universidade de Brasília \\ Instituto de Ciências Exatas \\ Departamento de Matemática
}

\title{
Grupos Finitos com um Grupo Metacíclico de Automorfismos
}

por

Emerson Ferreira de Melo

Brasília, 2015 
EMERSON FERREIRA DE MELO

Grupos Finitos com um Grupo Metacíclico de Automorfismos

Tese apresentada ao Departamento de Matemática da Universidade de Brasília, como parte dos requisitos para a obtenção do grau de doutor em matemática

\section{Tese de Doutorado}

Orientador: Prof. Dr. Pavel Shumyatsky

Brasilia 


\section{DEDICATÓRIA}

Dedico este trabalho à minha esposa, Lidiane, e ao meu filho, Arthur,

pelo amor que sempre me dedicaram

e pelo apoio incondicional.

A vocês o meu amor e reconhecimento eterno... 


\section{Agradecimentos}

Agradeço primeiramente a Deus por me permitir usufruir desta fase única da vida, que foi o doutorado.

À minha família, em especial à minha Mãe, ao Carlos, à Laura e ao Akio, por toda ajuda ao longo destes quatro anos.

Ao professor Pavel Shumyatsky pela sugestão do tema, pela orientação e por toda ajuda e incentivo nesta minha iniciação à pesquisa.

Ao professor Jhone Caldeira Silva pela sugestão e incentivo à minha vinda para o doutorado em Brasília.

Aos meus amigos, Mayer, Raimundo, Keidna, Alex, Ismael, Yerko, Renata, Ilana, Otto, Tailinny, Valdiego, Vínícios, Fábio, Lauro, Theo, Flávia, Enio, Eudes, Silvio, Agenor, Sunamita, Bruno, Jhoel, Kaliana, Daiane, Lucimeire, Cleilton, Kélem, Tarcísio, Wembesom. Devo muito a vocês.

Ao professor Evgeny Khukhro pela sugestão de alguns problemas estudados neste trabalho.

Agradeço aos professores participantes da banca Marinês Guerreiro, Jhone Caldeira Silva, Aline Gomes da Silva Pinto e Cristina Acciarri por aceitarem o convite e também pelas milhares de correções e sugestões.

Ao professor Gustavo Fernández Alcober pela orientação e hospitalidade durante minha visita à Universidad del País Vasco. Também aos amigos que fiz lá, em especial à Oihana, Mima, Ainhoa e ao Imanol pela receptividade e ajuda na adaptação.

Aos professores e funcionários do departamento de matemática e a todos que contribuíram para a realização deste trabalho.

À CAPES e ao CNPq pelo suporte financeiro. 


\section{Resumo}

Seja $M=F H$ um grupo finito o qual é um produto de dois subgrupos cíclicos $F$ e $H$, onde $F$ é um subgrupo normal e todos os elementos de $M \backslash F$ possuem ordem prima $p$. Suponha que $M$ age por automorfismos sobre um grupo finito $G$ de tal maneira que $C_{G}(F)=1$. Neste trabalho mostramos que propriedades de $G$ tais como comprimento de Fitting, nilpotência, expoente e leis positivas estão relacionadas com as respectivas propriedades dos subgrupos de pontos fixos dos elementos de $M \backslash F$. 


\section{Abstract}

Let $M=F H$ be a finite group which is a product of two cyclic subgroups $F$ and $H$, where $F$ is a normal subgroup and all elements of $M \backslash F$ have prime order $p$. Suppose that $M$ acts as a group of automorphisms on a finite group $G$ in such a manner that $C_{G}(F)=1$. In the present work we show that some properties of $G$ such as Fitting height, nilpotency, exponent and positive laws are related to the respective properties of the subgroups of fixed-points of elements in $M \backslash F$. 


\section{Lista de Símbolos}

$|G| \quad$ ordem de um grupo $G$

$\pi(G) \quad$ conjuntos dos números primos divisores de $|G|$

$C_{G}(A)$ centralizador de $A$ em $G$

$N_{G}(A)$ normalizador de $A$ em $G$

$\Phi(G) \quad$ subgrupo de Frattini de $G$

$G^{n} \quad$ subgrupo gerado pelo conjunto das $n$-ésimas potências dos elementos de $G$

$G^{(n)} \quad n$-ésimo termo da série derivada de $G$

$\gamma_{n}(G) \quad n$-ésimo termo da série central inferior de $G$

$D_{i}(G) \quad$ subgrupo de $G$ definido por $D_{i}(G)=\prod_{j p^{k} \geq i} \gamma_{j}(G)^{p^{k}}$

$O_{\pi}(G)$ produto dos $\pi$-subgrupos normais de $G$, onde $\pi$ é um conjunto de números primos

$F(G) \quad$ subgrupo de Fitting de $G$

$F_{n}(G) \quad n$-ésimo termo da série de Fitting de $G$

$h(G) \quad$ altura de Fitting de $G$ 


\section{Sumário}

$\begin{array}{ll}\text { Introdução } & 1\end{array}$

1 Preliminares $\quad 6$

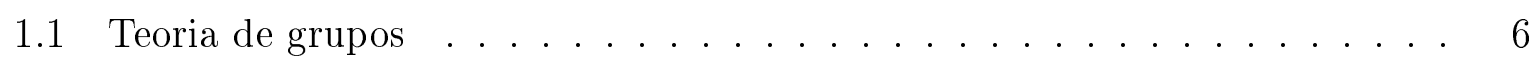

1.2 Introdução aos $p$-grupos potentes . . . . . . . . . . . . . 8

1.3 Leis positivas $\ldots \ldots \ldots \ldots \ldots \ldots$

1.4 Automorfismos de grupos ....................... 11

1.5 Grupos de Frobenius . . . . . . . . . . . . . . . . 14

1.6 Teoria de representações $\ldots \ldots \ldots \ldots \ldots$

1.7 Grupos metacíclicos . . . . . . . . . . . . . . 20

1.8 Álgebras de Lie . . . . . . . . . . . . . . . . . . 23

1.9 Álgebra de Lie associada a um $p$-grupo . . . . . . . . . . . . 26

2 Grupos metacíclicos como grupo de automorfismos 28

2.1 Grupos de Frobenius como grupos de automorfismos . . . . . . . . . 28

2.2 Grupos diedrais como grupos de automorfismos . . . . . . . . . . 30

2.3 Novos resultados . . . . . . . . . . . . . . . . . . . 31

3 Altura de Fitting de grupos finitos com um grupo metacíclico de automorfismos

3.1 Um resultado auxiliar sobre $\mathbb{F} G$-módulos $\ldots \ldots \ldots$. . . . . . . . 36 
3.2 Prova do teorema principal . . . . . . . . . . . . . . . . 43

4 Expoente e leis positivas de grupos finitos com um grupo metacíclico de automorfismos

4.1 Expoente de grupos finitos com um grupo metacíclico de automorfismos . . 47

4.2 Leis positivas em grupos finitos com um grupo diedral de automorfismos . 48

5 Considerações finais

Referências bibliográficas 


\section{Introdução}

Seja $G$ um grupo e $\varphi$ um elemento do grupo de automorfismos de $G$. Denotamos por $C_{G}(\varphi)$ o subgrupo formado pelos pontos fixos de $\varphi$ em $G$, que é

$$
C_{G}(\varphi)=\left\{x \in G ; x^{\varphi}=x\right\}
$$

O subgrupo $C_{G}(\varphi)$ também é chamado de centralizador de $\varphi$ em $G$. No caso particular em que $C_{G}(\varphi)=1$, dizemos que $\varphi$ é livre de pontos fixos em $G$.

De maneira análoga, dado um subgrupo $A$ do grupo de automorfismos de $G$, definimos o centralizador de $A$ em $G$ como $C_{G}(A)=\left\{x \in G ; x^{a}=x\right.$ para todo $\left.a \in A\right\}$ e dizemos que $A$ é livre de pontos fixos em $G$, sempre que $C_{G}(A)=1$.

É bem conhecido que propriedades do subgrupo $C_{G}(\varphi)$ possuem influência sobre a estrutura de todo o grupo $G$. Por exemplo, temos o famoso Teorema de Thompson [40] que diz que se um grupo finito $G$ admite um automorfismo de ordem prima $p$ livre de pontos fixos, então $G$ é nilpotente. Neste caso, por um resultado de Higman [17] sabemos que a classe de nilpotência de $G$ depende apenas do número primo $p$. No caso geral, onde $G$ admite um automorfismo livre de pontos fixos de ordem $n$, também sabemos pela classificação dos grupos simples [16] que $G$ deve ser solúvel, porém não está provado se o comprimento derivado depende apenas de $n$.

Em um caso mais geral, temos um resultado provado por Turull em [41], o qual diz que se um grupo solúvel finito $G$ admite um grupo de automorfismos $A$ tal que a ordem de $G$ é coprima com a ordem de $A$, então o comprimento de Fitting de $G$ é limitado apenas pelo comprimento de Fitting do subgrupo $C_{G}(A)$ e pelo número de primos cujo produto é $|A|$.

Entretanto, sabemos que existem parâmetros, tais como o expoente, que não podem ser limitados pela ordem e pelo respectivo parâmetro dos pontos fixos de um automorfismo. Por exemplo, para um grupo abeliano arbitrário de ordem ímpar, a função que leva cada 
elemento em seu inverso é um automorfismo livre de pontos fixos de ordem 2. Porém, neste caso específico do expoente, Khukhro e Shumyatsky em [19] provaram que se um grupo finito $G$ admite um grupo abeliano não cíclico de automorfismos $A$ tal que a ordem de $G$ é coprima com a ordem de $A$, então o expoente de $G$ depende de $|A|$ e do expoente dos subgrupos $C_{G}(a), a \in A \backslash\{1\}$. Tal exemplo ilustra o fato de que para obter informações sobre a estrutura de $G$, às vezes é necessário saber informações não apenas do subgrupo $C_{G}(A)$, e sim dos centralizadores de todos os elementos de $A$.

Recentemente, motivado pelo Problema 17.72 do Kourovka Notebook [44] proposto por Mazurov (veja abaixo), problemas onde um grupo de Frobenius $K B$ com núcleo $K$ e complemento $B$ age como grupo de automorfismos sobre um grupo finito $G$, de tal maneira que $C_{G}(K)=1$, vem sendo estudado. Lembre-se que um grupo de Frobenius $K B$ com núcleo $K$ e complemento $B$ pode ser definido como o produto semidireto de $K$ por $B$ tal que $C_{K}(b)=1$ para todo $b \in B \backslash\{1\}$.

Problema 0.0.1. 17.72 do Kourovka Notebook [44]] Seja KB um grupo de Frobenius com núcleo $K$ e complemento $B$. Suponha que $K B$ age sobre um grupo finito $G$ de tal modo que GK também seja um grupo de Frobenius com núcleo $G$ e complemento $K$.

a) A classe de nilpotêcia de $G$ é limitada em termos de $|B|$ e da classe de nilpotência de $C_{G}(B)$ ?

b) $O$ expoente de $G$ é limitado em termos de $|B|$ e do expoente de $C_{G}(B)$ ?

Em [33] Makarenko e Shumyatsky provaram uma resposta afirmativa para 17.72(a). O item $17.72(b)$ permanece sem resposta.

Relembramos que pelos teoremas de Thompson e de Higman citados anteriormente, o núcleo de um grupo de Frobenius é nilpotente de classe limitada pelo menor primo que divide a ordem do complemento. Também sabemos que os subgrupos de Sylow do complemento são cíclicos ou quatérnios generalizados, veja por exemplo [15, Teorema 10.3.1]. Sendo assim, no problema acima os grupo $G$ e $K$ são nilpotentes, pois são núcleos de grupos de Frobenius. Mais ainda, os subgrupos de Sylow de $K$ são cíclicos ou quatérnios generalizados, pois $K$ é complemento de $G K$. Por outro lado, como $K$ é nilpotente, seus subgrupos de Sylow são característicos, logo $K$ não possui um subgrupo quatérnio como subgrupo de Sylow, pois tal subgrupo possui centro de ordem 2, o qual seria fixado pelos elementos de $B$. Com isso, vemos que o Problema 0.0.1 pode ser reduzido para o caso onde $K$ é um grupo cíclico e, mais ainda, isso é suficiente para reduzir para o caso onde $K B$ é um grupo de Frobenius metacíclico e $|K|$ é um número primo. 
Em [25] Khukhro, Makarenko e Shumyatsky consideraram o caso mais geral onde a hipótese "GK é um grupo de Frobenius" do problema original, foi substituída por $C_{G}(K)=1$. Neste caso, eles provaram, por exemplo, que se um grupo finito $G$ admite um grupo de Frobenius $K B$ com núcleo $K$ e complemento $B$ tal que $C_{G}(K)=1$ e $C_{G}(B)$ é nilpotente, então $G$ é nilpotente e, apenas no caso particular onde $K$ é cíclico, a classe de nilpotência de $G$ é limitada apenas por $c$ e $|B|$. No mesmo trabalho eles provaram que outros parâmetros como posto e ordem de tal grupo $G$ estão relacionados com os respectivos parâmetros de $C_{G}(B)$ e com $|B|$. Além disso, para grupos de Frobenius com núcleo cíclico, provaram que o expoente de $G$ é limitado pelo expoente de $C_{G}(B)$ e por $|K B|$.

Um grupo de Frobenius com núcleo cíclico é necessariamente um grupo metacíclico (veja o comentário após o Teorema 1.6.5). Diante disso, é natural tentar estender os resultados provados para grupos de Frobenius metacíclicos como grupo de automorfismos para outras famílias de grupos metacíclicos. Neste sentido, Shumyatsky provou em [36] que se um grupo finito $G$ admite um grupo diedral $D=\langle\alpha, \beta\rangle$ gerado por duas involuções $\alpha$ e $\beta$ como grupo de automorfismos com $C_{G}(\alpha \beta)=1$, então, assim como para grupos de Frobenius, parâmetros como classe de nilpotência, posto e ordem de um tal grupo $G$ estão relacionados com os respectivos parâmetros de $C_{G}(\alpha)$ e $C_{G}(\beta)$. Lembre-se que um grupo diedral é Frobenius se, e somente se, a ordem de $\alpha \beta$ é ímpar.

O Problema 17.72 motivou vários outros resultados sobre grupos finitos e anéis de Lie admitindo um grupo de Frobenius como grupo de automorfismos onde o núcleo é cíclico, veja por exemplo [20, 21, 22, 23, 24, 25, 33]. Também motivou outros trabalhos onde o núcleo não é necessariamente cíclico, por exemplo [1, 4, 28].

Vale também mencionar que recentemente Ercan, Guloglu e Khukhro em [12, 13] consideraram o caso onde um grupo finito $G$ admite um grupo "Frobenius-like" como grupo de automorfismo. Um grupo $A R$ é dito Frobenius-like com núcleo $A$ e complemento $R$ se $A$ é um subgrupo normal nilpotente e $A R / A^{\prime}$ é um grupo de Frobenius com núcleo $A / A^{\prime}$ e complemento $R A^{\prime} / A^{\prime}$. Neste caso, eles também consideraram que $C_{G}(A)=1 \mathrm{e}$ obtiveram restrições sobre a estrutura de $G$ em função das propriedades de $C_{G}(R)$.

O objetivo deste trabalho é estudar grupos finitos admitindo a seguinte família de grupos metacíclicos de automorfismos: Seja $M=F H$ um grupo finito o qual é um produto de dois subgrupos cíclicos $F$ e $H$, onde $F$ é um subgrupo normal e todos os elementos de $M \backslash F$ possuem ordem prima $p$. Exemplos de tais grupos são:

1) os grupos de Frobenius com núcleo cíclico $F$ e complemento $H$ de ordem prima $p$; 
2) os grupos diedrais, onde usando a notação do parágrafo anterior, $F=\langle\alpha \beta\rangle$ e $H$ é o subgrupo gerado por um elemento fora de $F$.

Usando a identidade $\left(f h^{-1}\right)^{p}=f \cdot f^{h} \cdots \cdot f^{h^{p-1}} h^{-p}$, vemos que, de um modo geral, $M$ é o produto semidireto de um grupo cíclico $F$ por um grupo $H$ de ordem $p$, onde

$$
f \cdot f^{h} \cdots \cdot f^{h^{p-1}}=1
$$

para todos $f \in F$ e $h \in H \backslash\{1\}$. Como consequência temos que $Z(M)=C_{F}(H)$ possui expoente $p$.

Seja $M$ um grupo metacíclico da família descrita acima. Suponha que $M$ age como um grupo de automorfismos sobre um grupo finito $G$ de tal maneira que $C_{G}(F)=1$. Aqui mostramos que propriedades e parâmetros de $G$ tais como altura de Fitting, nilpotência, expoente e leis positivas estão relacionadas com as respectivas propriedades e parâmetros dos pontos fixos dos elementos de $M \backslash F$.

Recordamos que o subgrupo de Fitting $F(G)$ de um grupo finito $G$ é definido como o maior subgrupo normal nilpotente de $G$ e a série de Fitting de $G$ é definida do seguinte modo: fixamos $F_{0}(G)=1$ e definimos

$$
F_{i+1}(G) / F_{i}(G)=F\left(G / F_{i}(G)\right)
$$

para $i \geq 1$. Se $G$ é um grupo solúvel, o menor valor $h=h(G)$ tal que $G=F_{h}(G)$ é chamado de altura de Fitting de $G$.

O seguinte resultado foi apresentado em [9].

Teorema A. Seja $M=$ FH um grupo finito o qual é um produto de dois subgrupos cíclicos $F$ e $H$, onde $F$ é um subgrupo normal e todos os elementos de $M \backslash F$ possuem ordem prima p. Suponha que $M$ aja sobre um grupo finito $G$ de tal maneira que $C_{G}(F)=1$. Então $F_{i}\left(C_{G}(H)\right)=F_{i}(G) \cap C_{G}(H)$, para todo inteiro positivo $i$ e $h(G) \leq h\left(C_{G}(H)\right)+1$.

Como um corolário do teorema acima provamos no Capítulo 3 que se $M$ age sobre um grupo finito $G$ de tal maneira que $C_{G}(F)=1$ e $C_{G}(x)$ é nilpotente, para todo $x \in M \backslash F$, então $G$ é nilpotente.

No Capítulo 4 estudamos o expoente de grupos finitos admitindo um grupo metacíclico de automorfismo, onde provamos o seguinte resultado.

Teorema B. Seja $M=$ FH um grupo finito o qual é um produto de dois subgrupos cíclicos $F$ e $H$, onde $F$ é um subgrupo normal e todos os elementos de $M \backslash F$ possuem ordem prima p. Suponha que $M$ aja sobre um grupo finito $G$ de tal maneira que $C_{G}(F)=1$ e o 
expoente de $C_{G}(x)$ divide e para todos os elementos $x \in M \backslash F$. Então o expoente de $G$ é $(e,|M|)$-limitado.

Para o caso onde $M$ é um grupo diedral, provamos um caso mais geral do resultado anterior. O seguinte teorema foi apresentado em [8].

Teorema C. Seja $D=\langle\alpha, \beta\rangle$ um grupo diedral gerado por duas involuções $\alpha$ e $\beta$. Suponha que $D$ aja sobre um grupo finito $G$ de tal modo que $C_{G}(\alpha \beta)=1$ e ambos $C_{G}(\alpha)$ e $C_{G}(\beta)$ satisfazem uma lei positiva de grau $k$. Então $G$ satisfaz uma lei positiva de grau limitado somente por $k$ e $|D|$.

O presente trabalho está dividido em quatro capítulos. No primeiro capítulo apresentamos os resultados básicos da Teoria de Grupos e Álgebras de Lie utilizados neste trabalho. Em particular, na Seção 1.7 damos um descrição dos grupos metacíclicos considerados aqui. No segundo capítulo provamos resultados auxiliares sobre grupos finitos admitindo tais grupos metacíclicos de automorfismos, os quais são utilizados nos capítulos posteriores. No terceiro capítulo provamos o Teorema A e seus corolários. Finalmente, no Capítulo 4 provamos os Teoremas B e C. 


\section{Preliminares}

Neste capítulo apresentamos algumas definições e alguns resultados relacionados à Teoria de Grupos e Álgebras de Lie, os quais fazem parte do aparato teórico necessário às discussões deste trabalho.

\subsection{Teoria de grupos}

Dado um grupo $G$, definimos o comutador de dois elementos $x, y \in G$, por $[x, y]=$ $x^{-1} y^{-1} x y$ e definimos, por indução, o comutador de $r$ elementos $x_{1}, x_{2}, \ldots, x_{r} \in G$ por $\left[x_{1}, x_{2}, \ldots, x_{r}\right]=\left[\left[x_{1}, x_{2}, \ldots, x_{r-1}\right], x_{r}\right]$, onde $\left[x_{1}\right]=x_{1}$.

Agora, dados dois subconjuntos $A$ e $B$ de um grupo $G$, definimos o subgrupo $[A, B]$ como

$$
[A, B]=\langle[a, b]: a \in A \text { e } b \in B\rangle .
$$

De forma análoga à definição de comutador de $r$ elementos, dados $X_{1}, X_{2}, \ldots, X_{r}$ subconjuntos de $G$, definimos $\left[X_{1}, X_{2}, \ldots, X_{r}\right]$ como sendo o subgrupo de $G$ gerado por todos comutadores da forma $\left[x_{1}, x_{2}, \ldots, x_{r}\right]$, onde $x_{i} \in X_{i}$.

Definição 1.1.1. A série derivada de um grupo G é definida por:

$$
\begin{aligned}
G^{(0)} & =G ; \\
G^{(1)} & =[G, G] ; \\
& \vdots \\
G^{(n)} & =\left[G^{(n-1)}, G^{(n-1)}\right] .
\end{aligned}
$$

Um grupo é dito solúvel de comprimento derivado $n$ quando $n$ é o menor número natural tal que $G^{(n)}=1$. 
Definição 1.1.2. A série central inferior de um grupo $G$ é dada por:

$$
\begin{aligned}
\gamma_{1}(G) & =G ; \\
\gamma_{2}(G) & =[G, G] \\
& \vdots \\
\gamma_{s+1}(G) & =\left[\gamma_{s}(G), G\right] .
\end{aligned}
$$

Dizemos que um grupo é nilpotente de classe c quando c é o menor número natural tal que $\gamma_{c+1}(G)=1$.

De posse da definição de grupo nilpotente, temos os seguintes resultados, cuja demonstração pode ser encontrada em [27, Cap. 2].

Teorema 1.1.3. Dado um grupo $G$, as seguintes afirmações são equivalentes:

(i) $\gamma_{c+1}(G)=1$;

(ii) $\left[g_{1}, g_{2}, \ldots, g_{c+1}\right]=1$, para todo $g_{i} \in G$.

Definimos o centro $Z(G)$ de um grupo $G$ como

$$
Z(G)=\{z \in G \mid[z, g]=1 \text { para todo } g \in G\} .
$$

Teorema 1.1.4. Um grupo $G$ é nilpotente de classe $c>1$ se, e somente se, $G / Z(G)$ é nilpotente de classe $c-1$.

Agora, seja $X$ um subconjunto de um grupo $G$. Denotamos por $\langle X\rangle$ o subgrupo de $G$ gerado por $X$. No caso particular em que $X$ gera todo o grupo $G$, escrevemos $G=\langle X\rangle$.

Teorema 1.1.5. Se um grupo $G$ é gerado por um subconjunto $X$, então $G$ é nilpotente de classe $\leq c$ se, e somente se, todo comutador de peso $c+1$ com entradas em $X$ é igual $a 1$.

O próximo teorema será bastante útil. Embora, neste trabalho estejamos interessados em sua versão para álgebras de Lie, a qual será apresentada posteriormente.

Teorema 1.1.6 (Hall [18]). Seja $N$ um subgrupo normal de um grupo $G$. Se $N$ é nilpotente de classe $k$ e $G /[N, N]$ é nilpotente de classe c, então $G$ é nilpotente e sua classe de nilpotência é limitada por $(c-1) \frac{k(k+1)}{2}+k$.

Os seguintes resultados são bastante conhecidos na literatura.

Teorema 1.1.7 (Teorema 2.3.5 [15]). Seja G um grupo finito. Então G é nilpotente se, e somente se, $G$ é o produto direto de seus p-subgrupos de Sylow. 
Teorema 1.1.8 (Lema 6.1.1[15]). Se $N$ e $M$ são subgrupos normais nilpotentes de um grupo $G$, então o subgrupo NM é nilpotente.

Finalizamos esta seção com três definições importantes para este trabalho: as definições de subgrupo, série e altura de Fitting de um grupo finito.

Definição 1.1.9. Definimos o subgrupo de Fitting de um grupo finito $G$, o qual denotamos por $F(G)$, como o produto de todos os seus subgrupos normais nilpotentes.

Considere $r$ um número primo e $r^{\prime}$ o conjunto dos números primos diferentes de $r$. Vale ressaltar que o subgrupo de Fitting $F(G)$ de um grupo finito $G$ pode ser escrito como a interseção dos subgrupos $O_{r^{\prime}, r}(G)$, onde $r \in \pi(G)$ e $O_{r^{\prime}, r}(G)$ é a imagem inversa de $O_{r^{\prime}}\left(G / O_{r}(G)\right)$.

Definição 1.1.10. A série de Fitting de um grupo G é definida do seguinte modo: fixamos $F_{0}(G)=1$ e definimos

$$
F_{i+1}(G) / F_{i}(G)=F\left(G / F_{i}(G)\right),
$$

para $i=0,1, \ldots$ Se $G$ é um grupo solúvel, o menor valor $h=h(G)$ tal que $G=F_{h}(G)$ é chamado de altura de Fitting de $G$.

\subsection{Introdução aos $p$-grupos potentes}

Nosso principal objetivo aqui é apresentar alguns resultados e propriedades dos $p$ grupos potentes. Em particular, estamos interessados em resultados que garantem a existência de $p$-subgrupos potentes de "indice convenientemente limitado" em um p-grupo qualquer. Uma demonstração dos resultados desta seção pode ser encontrada em [10, Cap. 2] e [26, Cap. 11].

Nesta seção, $p$ sempre denota um número primo. Seja $G$ um $p$-grupo finito, definimos, para todo $i \geq 0$, os subgrupos característicos

$$
G^{p^{i}}=\left\langle x^{p^{i}} \mid x \in G\right\rangle
$$

O subgrupo de Frattini $\Phi(G)$ de um p-grupo finito $G$ é definido como a interseção de todos os seus subgrupos maximais. Vale relembrar que o subgrupo de Frattini é igual à $G^{p}[G, G]$ e, consequentemente, o grupo quociente $G / \Phi(G)$ é um $p$-grupo abeliano elementar.

Definição 1.2.1. Um p-grupo finito $G$ é dito potente se $p$ é ímpar e $G / G^{p}$ é abeliano, ou se p é par e $G / G^{4}$ é abeliano. 
Definição 1.2.2. Um subgrupo $N$ de um p-grupo finito $G$ é dito potentemente imerso em $G$, se p é ímpar e $[N, G] \leq N^{p}$, ou p é par e $[N, G] \leq N^{4}$.

Assim, $G$ é potente se, e somente se, $G$ é potentemente imerso em $G$ e, quando $p$ é ímpar, temos que $G$ é potente se, e somente se, $G^{p}=\Phi(G)$.

Definimos agora uma importante série de subgrupos característicos de um $p$-grupo, chamada p-série inferior.

Definição 1.2.3. Seja $G$ um p-grupo finito. Definimos a p-série inferior de $G$ da seguinte forma,

$$
P_{1}(G)=G, \quad \text { e } P_{i+1}(G)=P_{i}(G)^{p}\left[P_{i}(G), G\right], \quad \text { para } i>1 .
$$

Para simplificar a notação, escrevemos

$$
G_{i}=P_{i}(G)
$$

Lema 1.2.4 (Teorema $2.7[10]$ ). Seja $G=\left\langle a_{1}, \ldots, a_{d}\right\rangle$ um p-grupo potente. Para cada $i$ temos:

(i) $G_{i}$ é potentemente imerso em $G$ e $G_{i+1}=G_{i}^{p}=\Phi\left(G_{i}\right)$;

(ii) $G_{i}=G^{p^{i-1}}=\left\{x^{p^{i-1}} \mid x \in G\right\}=\left\langle a_{1}^{p^{i-1}}, \ldots, a_{d}^{p^{i-1}}\right\rangle$;

(iii) a aplicação $x \rightarrow x^{p}$ induz um homomorfismo de $G_{i} / G_{i+1}$ em $G_{i+1} / G_{i+2}$.

É interessante observar que o item (ii) do lema acima nos diz que se um p-grupo potente é gerado por elementos de ordem menor do que um valor $e$, então o expoente de tal grupo também é menor do que $e$. Essa propriedade de grupos potentes será usada no Capítulo 4 com o intuito de reduzir o problema de limitar o expoente de um $p$-grupo ao problema de encontrar um subgrupo potente de índice conveniente.

Para um $p$-grupo finito $G$ denotamos por $d(G)$ a quantidade mínima de um conjunto gerador de $G$. Assim $d(G)$ também é a dimensão de $G / \Phi(G)$ como um espaço vetorial sobre o corpo com $p$ elementos $\mathbb{F}_{p}$.

Teorema 1.2.5 (Teorema $2.9[10])$. Se $G$ é um p-grupo potente e $H \leq G$, então $d(H) \leq$ $d(G)$.

O posto de $G$, denotado por $r k(G)$, é definido como

$$
r k(G)=\sup \{d(H) \mid H \leq G\} .
$$


O teorema acima implica que se $G$ é um $p$-grupo potente, então $r k(G)=d(G)$.

Encerramos esta seção com um teorema que nos mostra que existe uma relação entre o posto de $G$ e o índice de alguns de seus subgrupos potentes. Outro resultado sobre a existência de $p$-subgrupos potentes será apresentado na seção sobre álgebras de Lie associadas a um p-grupo.

Teorema 1.2.6 (Teorema $2.13[10]$ ). Seja G um p-grupo finito de posto r. Então G possui um subgrupo potente característico de índice limitado por uma função que depende apenas de $p$ e r.

\subsection{Leis positivas}

O objetivo principal aqui é apresentar um resultado provado por Burns, Macedońska e Medvedev [3]. Este resultado permite entender um lei positiva qualquer, satisfeita por um grupo finito, em função de duas leis positivas mais "naturais": a lei $x^{e}$ e a lei de Malcev.

Sejam $F$ um grupo e $X$ um subconjunto de $F$. Dizemos que $F$ é um grupo livre com base X se, para todo grupo $G$, qualquer aplicação $f: X \rightarrow G$ pode ser estendida de modo único para um homomorfismo $\varphi: F \rightarrow G$. As demonstrações sobre a existência e construção de grupos livres podem ser encontradas em [35, Cap. 2]

Seja $F$ o grupo livre com base $X=\left\{x_{1}, x_{2}, \ldots\right\}$. Uma palavra positiva em $X$ é um elemento não trivial de $F$ não envolvendo os inversos dos elementos $x_{i}$, ou seja, são elementos da forma $x_{i_{1}}^{m_{1}} x_{i_{2}}^{m_{2}} \cdots x_{i_{k}}^{m_{k}}$, onde $k \geq 1$ e $m_{j} \geq 1$, para $j=1, \ldots, k$. Uma $l e i$ positiva de um grupo $G$ é uma identidade não trivial da forma $u \equiv v$, onde $u, v$ são palavras positivas em $F$, válida para toda substituição $X \rightarrow G$. O máximo dos comprimentos das palavras $u$ e $v$ é chamado de grau da lei $u \equiv v$. Um exemplo de lei positiva é $x y \equiv y x$, a qual é uma lei positiva de grau 2 satisfeita por todos os grupos abelianos.

Outro exemplo interessante é a lei positiva $x^{2} y^{2} \equiv y^{2} x^{2}$, a qual é satisfeita por todos os grupos que são extensões de um grupo abeliano por um grupo de expoente 2, como por exemplo os grupos diedrais. Tal exemplo mostra que parâmetros como classe de nilpotência e expoente não podem ser limitados pelo grau da lei, pois os grupos diedrais de ordem $2^{n}$ podem ter classe de nilpotência e expoente arbitrariamente grandes.

Temos ainda a lei de Malcev [32] $M_{c}(x, y)$ de duas variáveis e de grau $2^{c}$ definida pela relação $\alpha_{c}(x, y)=\beta_{c}(x, y)$, onde $\alpha_{c}$ e $\beta_{c}$ são definidos por $\alpha_{0}(x, y)=x, \beta_{0}(x, y)=y$ e a relação recursiva

$$
\alpha_{c}=\alpha_{c-1} \beta_{c-1} \quad \text { e } \beta_{c}=\beta_{c-1} \alpha_{c-1} .
$$


Assim, $M_{1}(x, y)$ é a lei apresentada acima $x y \equiv y x$ e $M_{2}(x, y)$ é a lei $x y y x \equiv y x x y$. É sabido que a lei de Malcev $M_{c}(x, y)$ é satisfeita por todos os grupos nilpotentes de classe c. Como consequência, um grupo que é uma extensão de um grupo nilpotente de classe $c$ por um grupo de expoente $e$ satisfaz a lei positiva $M_{c}\left(x^{e}, y^{e}\right)$.

De um modo geral, um grupo satisfazendo uma lei positiva não é necessariamente uma extensão de um grupo nilpotente por um grupo de expoente finito, isto foi demonstrado por Ol'shanskii e Storozhev [34]. Porém, temos o seguinte teorema:

Teorema 1.3.1 (Teorema 1 - Burns, Macedońska e Medvedev [3]). Existem funções c(n) e $e(n)$ dependendo somente de $n$ tais que qualquer grupo finito satisfazendo uma lei positiva de grau n é extensão de um grupo nilpotente de classe no máximo $c(n)$ por um grupo de expoente dividindo e $(n)$.

Na verdade, em [3] está provado que o teorema acima vale não apenas para grupos finitos, mas para uma classe de grupos maior, a qual inclui os grupos solúveis e residualmente finitos.

\subsection{Automorfismos de grupos}

Apresentaremos agora alguns resultados sobre automorfismos de grupos cujas demonstrações podem ser encontradas em [15, Cap. 10], [26, Cap. 1] e [27, Cap. 1].

Relembramos que um grupo $A$ age sobre um conjunto $\Omega$ se cada elemento $a \in A$ corresponde a uma bijeção $\pi_{a}: \Omega \longrightarrow \Omega$ de $\Omega$ de tal modo que $\pi_{a b}$ seja a composição de $\pi_{a}$ e $\pi_{b}$. Em outras palavras, a função $a \longrightarrow \pi_{a}$ é um homomorfismo de $A$ no grupo de permutações dos elementos de $\Omega$. E dizemos que a ação é fiel quando seu núcleo é igual a $1 \in A$.

Ao longo deste trabalho dizemos que um grupo $A$ age por automorfismos sobre um grupo $G$ quando a ação de $A$ sobre o conjunto $\Omega=G$ é um homomorfismo de $A$ em $A u t(G)$, onde $\operatorname{Aut}(G)$ é o grupo formado por todos automorfismos de $G$. Podemos também dizer simplesmente que $A$ age sobre $G$. Vemos que se tal ação é fiel, então $A$ pode ser identificado como um subgrupo de $\operatorname{Aut}(G)$.

Sejam $G$ um grupo e $H$ um subconjunto de $A u t(G)$. Dizemos que um subconjunto $X$ de $G$ é $H$-invariante se tivermos $X^{h} \subseteq X$, para todo $h \in H$, onde $X^{h}$ é a imagem do subconjunto $X$ pelo automorfismo $h$. Além disso, denotamos o subgrupo formado pelos pontos fixos de $H$ em $G$ por

$$
C_{G}(H)=\left\{g \in G \mid g^{h}=g, \text { para todo } h \in H\right\} .
$$


O subgrupo $C_{G}(H)$ também será chamado de centralizador de $H$ em $G$. Quando temos $C_{G}(H)=1$ dizemos que $H$ é livre de pontos fixos (ou age sem pontos fixos) em $G$.

Lema 1.4.1. Seja p um número primo. Se A é um p-grupo de automorfismos de um p-grupo finito não trivial $G$, então $C_{G}(A) \neq 1$.

Dados um elemento $\varphi \in \operatorname{Aut}(G)$ e um subgrupo normal $\varphi$-invariante $N$, podemos construir um automorfismo sobre o grupo quociente $G / N$ de modo natural: $(g N)^{\varphi}=g^{\varphi} N$. No decorrer do texto tal automorfismo induzido será denotado pelo mesmo símbolo $\varphi$. Além disso, do mesmo modo, dado um grupo de automorfismos $A$ de $G$ podemos construir um grupo de automorfismos sobre o grupo quociente $G / N$ onde $N$ é um subgrupo normal $A$-invariante.

O próximo lema nos permite entender, sob certas hipóteses, a relação entre os pontos fixos de um grupo de automorfismos e os pontos fixos do grupo de automorfismos induzido.

Lema 1.4.2. Suponha que um grupo finito $A$ age sobre um grupo finito $G$ e seja $N$ um subgrupo normal $A$-invariante de $G$ tal que $(|A|,|N|)=1$. Então $C_{G / N}(A)=C_{G}(A) N / N$.

\section{Automorfismos livres de pontos fixos}

Agora apresentamos alguns resultados que dizem respeito apenas a grupos finitos admitindo um automorfismo ou um grupo de automorfismos livres de pontos fixos.

Lema 1.4.3. Seja $G$ um p-grupo finito admitindo um grupo de automorfismos nilpotente A tal que $C_{G}(A)=1$. Seja $B$ o $p^{\prime}$-subgrupo maximal de $A$. Então $C_{G}(B)=1$.

Demonstração. Temos que o p-grupo $A / B$ age de modo natural sobre o p-grupo $C_{G}(B)$, e os pontos fixos desta ação são pontos fixos do grupo $A$. Logo, pelo Lema 1.4.1, devemos ter $C_{G}(B)=1$.

O próximo lema nos mostra uma maneira, muitas vezes conveniente, de escrever os elementos de um grupo finito admitindo um automorfismo sem pontos fixos. Além disso, ele nos fornece uma identidade, que depende do automorfismo, satisfeita por tais grupos.

Lema 1.4.4 (Lema 10.1.1 [15]). Seja $\varphi$ um automorfismo de ordem $n$ livre de pontos fixos de um grupo finito G. Então:

(i) todo elemento de $G$ pode ser escrito na forma $x^{-1} x^{\varphi}$ e $x^{\varphi} x^{-1}$, para apropriados $x \in G$. 
(ii) para todo $x \in G$, temos $x x^{\varphi} \cdots x^{\varphi^{n-1}}=x^{\varphi^{n-1}} \cdots x^{\varphi} x=1$.

É interessante comparar o seguinte lema com o Lema 1.4.2, uma vez que ambos descrevem os pontos fixos do automorfismo induzido. No entanto, no próximo lema não exigimos que a ordem do automorfismo seja coprima com a ordem do subgrupo invariante.

Lema 1.4.5 (Lema 10.1.3 [15]). Seja $\varphi$ um automorfismo livre de pontos fixos de um grupo finito $G$ e seja $N$ um subgrupo normal $\varphi$-invariante de $G$. Então $\varphi$ induz um automorfismo livre de pontos fixos em $G / N$.

Outra importante propriedade dos automorfismos sem pontos fixos é que deixam invariante apenas um $p$-subgrupo de Sylow, para cada primo em $\pi(G)$.

Lema 1.4.6 (Lema 10.1.2 [15]). Se $\varphi$ é um automorfismo livre de pontos fixos de G, então $\varphi$ deixa invariante somente um p-subgrupo de Sylow, para cada primo p divisor da ordem de $G$.

Os próximos dois resultados dizem respeito a grupos admitindo um automorfismo de ordem prima sem pontos fixos. Eles juntos dizem que se um grupo finito admite um automorfismo de ordem prima $p$ sem pontos fixos, então tal grupo é nilpotente de classe limitada apenas em termos de $p$.

Teorema 1.4.7 (Higman [17]). Se G é um grupo nilpotente admitindo um automorfismo livre de pontos fixos de ordem prima p, então a classe de nilpotência de $G$ é limitada por uma função $h(p)$ que depende apenas de $p$.

Teorema 1.4.8 (Thompson [40]). Se G admite um automorfismo livre de pontos fixos de ordem prima, então $G$ é nilpotente.

É importante ressaltar que é possível construir exemplos de grupos não nilpotentes admitindo um automorfismo livre de pontos fixos. Um exemplo pode ser encontrado em [15, pag. 336]. Por outro lado, como um corolário da classificação dos grupos simples finitos temos que um grupo finito admitindo um automorfismo sem pontos fixos é solúvel, veja por exemplo [16]. Diante disso, temos o seguinte teorema.

Teorema 1.4.9. Se G admite um automorfismo livre de pontos fixos, então G é solúvel.

De um modo geral, no teorema acima não sabemos se o comprimento derivado de $G$ pode ser limitado em função da ordem do automorfismo. Entretanto, vale o seguinte resultado para o comprimento de Fitting.

Teorema 1.4.10 (Dade [7]). Se G admite um automorfismo livre de pontos fixos de ordem $n$, então a altura de Fitting de $G$ é limitado por uma função que depende apenas de $n$. 


\subsection{Grupos de Frobenius}

A seguir apresentamos propriedades básicas de uma importante "classe" de grupos finitos, os chamados Grupos de Frobenius. O estudo de tais grupos motivaram inúmeros avanços na teoria dos grupos finitos, tais como a classificação dos grupos cujos subgrupos de Sylow são cíclicos ou quatérnio generalizado, o Teorema de Thompson sobre grupos com um automorfismo sem pontos fixos e etc.. Mais informações sobre esse interessante assunto podem ser encontradas ao longo de todo o Livro [15].

Seja $H$ um subgrupo de um grupo finito $G$. Considere $\Omega=\left\{H x_{i} \mid x_{i} \in G, 1 \leq i \leq n\right\}$ o conjunto formado pelas classes laterais de $H$ em $G$. Para cada $x \in G$ podemos definir uma permutação $\pi_{x}$ do conjunto $\Omega$ do seguinte modo

$$
\pi_{x}\left(H x_{i}\right)=H x_{i} x \text {, para cada } 1 \leq i \leq n .
$$

É sabido que a aplicação $\pi_{H}$ que associa a cada $x \in G$ a permutação $\pi_{x}$ é um homomorfismo de $G$ no grupo de permutação do conjunto $\Omega$, o qual podemos identificar com o grupo simétrico $S_{n}$. O núcleo do homomorfismo $\pi_{H}$ é o conjunto

$$
N=\left\{x \in G \mid x \in x_{i}^{-1} H x_{i} \text { para todo } i, 1 \leq i \leq n\right\} .
$$

Chamamos $\pi_{H}$ de representação transitiva de $G$ sobre o grupo de permutações das classes (a esquerda) de $H$. O caso em que $H=1$ é chamado de representação regular. De acordo com a definição anterior, vemos que o grupo $G / N$ age transitivamente sobre o conjunto $\Omega$, ou seja, dado dois elementos $H x_{l}$ e $H x_{m}$ em $\Omega$, existe $\bar{g} \in G / N$ tal que $\pi_{\bar{g}}\left(H x_{l}\right)=H x_{m}$. Mais ainda, vemos que o subgrupo $\pi_{H}\left(H^{x_{i}}\right)$ é o subgrupo que fixa o elemento $H x_{i}$ e que na representação regular somente o elemento neutro de $G$ fixa mais de um elemento de $\Omega$.

Definição 1.5.1. Dado um grupo $G$, dizemos que um subgrupo $H$ é disjunto de seus conjugados se $H \cap H^{x}=H$ ou $H \cap H^{x}=1$, para todo $x \in G$.

Usando a definição do homomorfismo $\pi_{H}$, vemos que $H$ é disjunto de seus conjugados se $\pi_{H}(H)$ fixa apenas os elementos $\left\{H x_{i}\right\}$, onde $x_{i}$ normaliza $H$.

Definição 1.5.2 (Grupo de Frobenius). Seja $H$ um subgrupo não trivial de um grupo finito $G$. Dizemos que $G$ é um grupo de Frobenius com complemento $H$ se $H$ é disjunto de seus conjugados e é seu próprio normalizador em $G$.

Em outras palavras, um grupo de Frobenius pode ser visto como um grupo de permutações de um conjunto $\Omega$ (as classe de $H$ ) onde somente a identidade fixa mais do que 
um elemento, mas os subgrupos que fixam um elemento de $\Omega$ são não triviais (a saber, $\pi_{H}\left(H^{x_{i}}\right)$ fixa somente o elemento $\left.H x_{i}\right)$.

O próximo teorema é devido a Frobenius.

Teorema 1.5.3 (Teorema 4.5.1 [15]). Seja G um grupo de Frobenius com complemento H. Considere o conjunto $\Omega=\left\{H x_{i} \mid x_{i} \in G, 1 \leq i \leq n\right\}$ das classe laterais de $H e$ o homomorfismo $\pi_{H}$. Então o subconjunto de $G$ consistindo da identidade junto com os elementos $x$ tais que $\pi_{x}$ não fixam nenhum elemento de $\Omega$ formam um subgrupo normal $K$ de $G$ de ordem igual ao indice de $H$ em $G$.

O subgrupo $K$ descrito no teorema anterior é chamado de núcleo de Frobenius de $G$. Além disso, temos

$$
K=\{1\} \cup\left(G \backslash \bigcup_{g \in G} H^{g}\right)
$$

O próximo resultado, em um certo sentido, nos permite construir grupos de Frobenius a partir do grupo de automorfismos de um grupo finito. Também nos mostra uma relação entre os grupo de Frobenius e os automorfismos sem pontos fixos.

Teorema 1.5.4. Sejam B um grupo finito e $A$ um subgrupo de Aut(B). Suponha que para todo elemento não trivial de $a \in A$ temos $C_{B}(a)=1$. Então o produto semidireto $G=B \rtimes A$ de $B$ por $A$ é um grupo de Frobenius com núcleo $B$ e complemento $A$.

Demonstração. Primeiro mostramos que $A$ é seu próprio normalizador. Note que dado $x \in G$ temos $A^{x}=A^{b}$, para algum $b \in B$, pois $G=B A$. Suponha que $A^{b}=A$, para algum $b \in B$. Então $[a, b] \in A$, para todo $a \in A$. Por outro lado, como $B$ é normal em $G$, temos que $[a, b] \in B$. Sendo assim, como $B \cap A=1$, temos $[a, b]=1$, para todo $a \in A$. Mas isso significa $b^{a}=b$, para todo $A$, ou seja, $b \in C_{B}(A)=1$.

Agora, precisamos mostrar que $A \cap A^{b}=A$ ou $A \cap A^{b}=1$, para todo $b \in B$. Suponha que $A \cap A^{b} \neq 1$ e fixe $a \in A \cap A^{b}$. Então $[a, b] \in A \cap B=1$. Portanto, $b \in C_{B}(a)=1 \mathrm{e}$ temos $A \cap A^{b}=A$.

Encerramos esta seção com um teorema que lista as principais propriedades de um grupo de Frobenius, sua demostração pode ser encontrada em [15, Teoremas 2.7.6 e 10.3.1]. 
Teorema 1.5.5. Seja $G$ um grupo de Frobenius com complemento $H$ e núcleo $K$. Então:

(i) $G=K H$ com $H \cap K$, ou seja, $G$ é o produto semidireto de $K$ por $H$;

(ii) $|H|$ divide $|K|-1$;

(iii) Todo elemento de $H \backslash\{1\}$ induz por conjugação um automorfismos de $K$ o qual fixa somente o elemento identidade de $K$;

(iv) $C_{G}(y) \subseteq K$, para todo elemento não trivial $y \in K$.

(v) $K$ é nilpotente e é abeliano se $|H|$ é par.

(vi) Os p-subgrupos de Sylow de H são cíclicos, se p é ímpar, e são cíclicos ou quatérnios generalizados se $p=2$.

(vii) Qualquer subgrupo de $H$ de ordem pq, onde $p$ e q são primos, é cíclico.

(viii) Se $|H|$ é ímpar, então $H$ é metacíclico, enquanto que se $|H|$ é par, $H$ possui um único elemento de ordem dois, o qual necessariamente pertence ao centro.

\subsection{Teoria de representações}

Discutimos aqui apenas alguns fatos básicos da Teoria de Representações Lineares. Nosso objetivo principal é apresentar o Teorema de Clifford, o qual será usado no Capítulo 3.

Sejam $\mathbb{F}$ um corpo e $V$ um espaço vetorial de dimensão finita sobre $\mathbb{F}$ e $G L(V)$ o grupo de transformações lineares invertíveis de $V$. Dado um grupo $G$, um homomorfismo $\rho: G \rightarrow G L(V)$ é dito uma representação linear de $G$ sobre $V$. O núcleo $K$ de $\rho$ é chamado de núcleo da representação. Se o homomorfismo $\rho$ é injetivo, dizemos $\rho$ é uma representação fiel de $G$.

Duas representações $\rho$ e $\nu$ de $G$ sobre $V$ são ditas equivalentes se existe um isomorfismo $\phi: V \rightarrow V$ tal que $\rho(g) \phi=\phi \nu(g)$, para todo $g \in G$.

A dimensão de $V$ sobre $\mathbb{F}$ é dita grau da representação $\rho$. Uma representação de grau 1 é chamada de representação afim. Neste trabalho nos restringimos apenas às representações de grau finito.

Se $W$ é um subespaço de $V$ invariante por $\rho(G)$, então claramente $\rho$ induz uma representação de $G$ sobre $W$, a qual chamamos de restrição de $\rho$ em $W$ e denotamos por $\left.\rho\right|_{W} \cdot$ 
Uma representação $\rho$ de $G$ sobre $V$ é dita irredutivel se 0 e $V$ são os únicos subespaços $\rho(G)$-invariantes de $V$. Caso contrário, dizemos que $\rho$ é redutivel. Além disso, dizemos que uma representação $\rho$ de $G$ sobre $V$ é completamente redutível quando

$$
V=V_{1} \oplus V_{2} \oplus \cdots V_{r}
$$

onde cada $V_{i}$ é um subespaço $\rho(G)$-invariante não trivial de $V$ e $\left.\rho\right|_{V_{i}}$ é irredutível.

Às vezes é mais conveniente tornar o espaço vetorial $V$ um $\mathbb{F} G$-módulo à esquerda definindo $g v=\rho(g)(v)$, para todo, $g \in G$ e $v \in V$. Feito isto, podemos usar a linguagem de módulos em vários conceitos que já definimos. Assim, por exemplo, um subespaço $\rho(G)$-invariante se torna um $\mathbb{F} G$-submódulo de $V$ e $V$ é $\mathbb{F} G$-módulo irredutível se 0 e $V$ são os únicos $\mathbb{F} G$-submódulos de $V$. Além disso, é interessante notar que cada elemento de $G$ se torna uma transformação linear de $V$, assim conceitos como os de autovalor e autovetor também podem ser usados.

Iniciamos apresentando alguns resultados básicos da teoria de representação de grupos abelianos. Uma demonstração dos resultados apresentados aqui pode ser encontrada em [15, Cap. 3].

Lema 1.6.1. Seja $V$ um $\mathbb{F} G$-módulo, onde $V$ é espaço vetorial sobre $\mathbb{F}$. Se um elemento $z \in Z(G)$ possui um autovalor $\lambda$ em $\mathbb{F}$, então $z v=\lambda v$, para todos $v \in V$. Em particular, se $V$ é um $G$-módulo fiel, então $z=1$ ou $\lambda \neq 1$.

O lema acima é particularmente importante no estudo de representação de grupos abelianos sobre um corpo algebricamente fechado, pois diz que todo elemento se comporta como uma transformação escalar. Ele também é usado para demonstrar os seguintes teoremas.

Teorema 1.6.2. Se $\rho$ é uma representação irredutivel de um grupo abeliano $G$ com núcleo $K$, então $G / K$ é cíclico. Em particular, um grupo abeliano nãa cíclico não possui uma representação irredutivel e fiel.

Teorema 1.6.3. Seja $G$ um grupo abeliano de ordem $n$ e $\mathbb{F}$ um corpo que contém a n-ésima raiz da unidade. Então toda representação irredutivel de $G$ sobre $\mathbb{F}$ é afim.

O teorema seguinte nos dá um critério suficiente para uma representação ser completamente irredutível.

Teorema 1.6.4 (Maschke - Teorema 3.3.1 [15]). Seja $\rho$ uma representação de G sobre $V$, onde $V$ é um espaço vetorial sobre $\mathbb{F}$. Assuma que $\mathbb{F}$ é de característica 0 ou de característica coprima com $|G|$. Então $\rho$ é completamente redutivel. 
Uma aplicação dos resultados acima para a Teoria de Grupos é o teorema seguinte:

Teorema 1.6.5. Seja $Q$ um q-grupo abeliano elementar e seja $P$ um p-subgrupo abeliano elementar não cíclico de $\operatorname{Aut}(Q)$, onde $p \neq q$. Então

$$
Q=\prod_{x \in P \backslash\{1\}} C_{Q}(x) .
$$

O teorema acima pode ser usado para provar que um grupo de Frobenius $K B$ com núcleo cíclico $K$ e complemento $H$ é necessariamente metacíclico, pois nesse casso $H$ deve ser abeliano e, como todo elemento do complemento age sem pontos fixos o teorema acima implica que $H$ deve ser cíclico.

Finalizamos esta seção com o Teorema de Clifford, cuja demonstração pode ser encontrada em [15, Teorema 3.4] . Dados uma representação irredutível de um grupo $G$ sobre $V$ e um subgrupo normal $H$ de $G$, ele nos permite saber como o espaço $V$ se decompõe em relação a ação de $H$. Vale também ressaltar que neste trabalho, especificamente no Capítulo 3, estamos mais interessados no item (iii) do Teorema de Clifford.

Teorema 1.6.6 (Clifford). Sejam $V$ um G-módulo irredutível e $H$ um subgrupo normal de $G$. Então $V$ é uma soma direta de subespaços $H$-invariantes $V_{i}, 1 \leq i \leq r$, os quais satisfazem as seguintes condições:

(i) $V_{i}=X_{i 1} \oplus X_{i 2} \oplus \cdots \oplus X_{i t}$, onde cada $X_{i j}$ é um $H$-submódulo irredutivel, $1 \leq i \leq r, t$ é independente de $i$, e $X_{i j}, X_{i^{\prime} j^{\prime}}$ são $H$-submódulos isomorfos se e somente se $i=i^{\prime}$.

(ii) Para qualquer H-submódulo $U$ de $V$, temos $U=U_{1} \oplus U_{2} \oplus \cdots \oplus U_{r}$, onde $U_{i}=$ $U \cap V_{i}, 1 \leq i \leq r$. Em particular, qualquer $H$-submódulo irredutível de $V$ está contido em algum dos $V_{i}$.

(iii) Para cada $x \in G$, a aplicação $\Pi(x): V_{i} \mapsto x V_{i}, 1 \leq i \leq r$ é uma permutação do conjunto $S=\left\{V_{1}, V_{2}, \ldots, V_{r}\right\}$ e $\Pi$ induz um representação transitiva de $G$ sobre $S$ por grupo de permutações. Além disso, o subgrupo $H C_{G}(H)$ está contido no núcleo de $\Pi$.

\section{Produto tensorial}

Em certas circunstâncias, demonstrações que envolvem as representações lineares se tornam mais fáceis se o corpo $\mathbb{F}$ contém uma raiz $m$-ésima da unidade, para $m$ conveniente, ou até mesmo que seja algebricamente fechado. A fim de contornar esse tipo de problema, 
apresentamos o conceito de Produto Tensorial e alguns aspectos básicos desta teoria. Maiores detalhes sobre este assunto podem ser encontrados em [27].

Sejam $A$ e $B$ dois espaços vetoriais sobre um corpo $\mathbb{F}$. O produto tensorial $A \otimes_{\mathbb{F}} B$ é definido como um par $(W, \psi)$, onde $W$ é um espaço vetorial sobre $\mathbb{F}$ e $\psi: A \times B \rightarrow W$ é uma aplicação bilinear que satisfaz a seguinte propriedade universal: para todo espaço vetorial $W^{\prime}$ sobre $\mathbb{F}$ e para toda aplicação bilinear $\phi: A \times B \rightarrow W^{\prime}$, existe uma única $T: W \rightarrow W^{\prime}$ linear tal que $\phi(u, v)=T(\psi(u, v))$ para todos $u \in A$ e $v \in B$. As seguintes propriedades valem para o produto tensorial $A \otimes_{\mathbb{F}} B$ :

$$
\begin{gathered}
r(a \otimes b)=r a \otimes b=a \otimes r b, \\
a \otimes\left(b_{1}+b_{2}\right)=a \otimes b_{1}+a \otimes b_{2}, \\
\left(a_{1}+a_{2}\right) \otimes b=a_{1} \otimes b+a_{2} \otimes b,
\end{gathered}
$$

onde $r \in \mathbb{F} ; a, a_{1}, a_{2} \in A ; b, b_{1}, b_{2} \in B$.

O seguinte exemplo de produto tensorial será usado no Capítulo 3. Considere $\mathbb{F}_{p} \mathrm{O}$ corpo com $p$ elementos. Sejam $V$ um espaço vetorial sobre $\mathbb{F}_{p}$ e $k$ um corpo de decomposição de algum polinômio sobre $\mathbb{F}_{p}$. Sabemos que o corpo $k$ também pode ser considerado como um espaço vetorial sobre $\mathbb{F}_{p}$. Sendo assim, podemos definir o produto tensorial $V \otimes_{\mathbb{F}_{p}} k$.

É interessante observar que o produto tensorial $V \otimes_{\mathbb{F}_{p}} k$ pode ser considerado como um espaço vetorial sobre $k$, definindo o produto por um elemento $\beta \in k \operatorname{como} \beta(a \otimes b)=$ $(a \otimes \beta b)$. Além disso, dado um automorfismo $\varphi$ de $V$, podemos considerar $\varphi$ como um automorfismo de $V \otimes_{\mathbb{F}_{p}} k$ fazendo $\varphi$ agir de forma trivial sobre $k$, ou seja, $(a \otimes b)^{\varphi}=a^{\varphi} \otimes b$, para todo $a \otimes b \in V \otimes_{\mathbb{F}_{p}} k$.

O próximo resultado nos dá uma relação entre os pontos fixos de $\varphi$ em $V$ e os pontos fixos de $\varphi$ em $V \otimes_{\mathbb{F}_{p}} k$.

Lema 1.6.7. Considerando $\widetilde{V}=V \otimes_{\mathbb{F}_{p}} k$ o produto tensorial definido acima e $\varphi$ um automorfismo de $V$ agindo sobre $\widetilde{V}$ da forma descrita anteriormente, temos

$$
C_{\widetilde{V}}(\varphi)=C_{V}(\varphi) \otimes_{\mathbb{F}_{p}} k
$$

Encerramos esta seção com um teorema que relaciona as representação sobre $V$ e as representações sobre $V \otimes_{\mathbb{F}_{p}} k$.

Teorema 1.6.8 (Teorema 29.7 - Noether, Deuring [6]). Sejam $V$ um espaço vetorial de dimensão finita sobre um corpo $\mathbb{F}$ e $k$ uma extensão de $\mathbb{F}$. Se duas representações $\rho$ e $\nu$ de $V$ são equivalentes em $\widetilde{V}=V \bigotimes_{\mathbb{F}} k$, então $\rho$ e $\nu$ são equivalentes como representações de $V$. 


\subsection{Grupos metacíclicos}

Um grupo $M$ é dito metacíclico quando contém um subgrupo normal cíclico $F$ tal que $M / F$ também é cíclico. É imediato verificar que o produto semidireto de grupos cíclicos são exemplos de grupos metacíclicos. No entanto, o grupo dos quatérnios $Q_{8}$ é um exemplo de grupo metacíclico que não é um produto semidireto de subgrupos cíclicos. No caso especial onde $M$ possui um subgrupo $H$ isomorfo a $M / F$ tal que $M=F \rtimes H$ dizemos que $M$ é extensão cindida de $F$ por $H$.

Em geral, um grupo metacíclico $M$ é gerado por dois elementos e possui a seguinte apresentação

$$
M=\left\langle a, b \mid a^{n}=1, b^{m}=a^{u}, a^{b}=a^{s}\right\rangle,
$$

onde $n, m$, u e $s$ são inteiros positivos que satisfazem $s^{m} \equiv 1(\bmod n)$ e $u(s-1) \equiv 0(\bmod n)$. Outra observação interessante é que o grupo derivado $M^{\prime}$ de $M$ é cíclico, mas não é verdade em geral que o grupo quociente $M / M^{\prime}$ seja cíclico, por exemplo $Q_{8} /\left(Q_{8}\right)^{\prime}$ é um 2-grupo abeliano elementar de posto 2.

\section{Grupos diedrais}

Um família de grupos metacíclicos, particularmente importante para este trabalho, são os grupos diedrais, cuja apresentação é:

$$
D_{n}=\left\langle h, f \mid h^{2}=f^{n}=1, f^{h}=f^{-1}\right\rangle .
$$

O lema seguinte nos diz que na verdade os grupos diedrais são os grupos gerados por dois elementos de ordem dois. Neste trabalho chamamos de involuções os elementos de ordem dois de um grupo.

Lema 1.7.1. Seja $D$ um grupo finito gerado por duas involuções $\alpha$ e $\beta$. Então $D$ é um grupo diedral.

Demonstração. Como $\alpha$ e $\beta$ são involuções temos que $\beta \alpha$ é o inverso de $\alpha \beta$. Consequentemente $(\alpha \beta)^{\alpha}=\beta \alpha=(\alpha \beta)^{-1}$. Em outras palavras, $\alpha$ leva cada elemento do subgrupo $\langle\alpha \beta\rangle$ em seu inverso. Por outro lado, vemos que $D$ também é gerado por $\alpha$ e $\alpha \beta$, ou seja, $D$ é um grupo diedral. 
Como uma consequência do lema acima podemos apresentar um grupo diedral da seguinte forma

$$
D_{n}=\left\langle\alpha, \beta \mid \alpha^{2}=\beta^{2}=(\alpha \beta)^{n}=1\right\rangle .
$$

Além disso, como $\alpha$ leva cada elemento de $F=\langle\alpha \beta\rangle$ em seu inverso, vemos que se $|\alpha \beta|$ é ímpar, então $\alpha$ age sobre $F$ sem ponto fixos, consequentemente, pelo Lema 1.5.4, o grupo $D_{n}$ é um grupo de Frobenius com núcleo $F$ e complemento $\langle\alpha\rangle$.

Seja $D=\langle\alpha, \beta\rangle$ um grupo diedral gerado por duas involuções $\alpha$ e $\beta$. O próximo lema diz que $\alpha$ e $\beta$ são conjugados se, e somente se, $D$ é um grupo de Frobenius.

Lema 1.7.2. Seja $D$ um grupo diedral gerado por duas involuções $\alpha$ e $\beta$. Então $\alpha$ e $\beta$ são conjugados se, e somente se, $|\alpha \beta|$ é ímpar.

Demonstração. Fixe $f=\alpha \beta$. Assim, temos $D=\langle\alpha, f\rangle$ e $\beta=\alpha f$. Mais ainda, vemos que é suficiente verificar se $\alpha$ e $\beta$ são conjugados por um elemento em $\langle f\rangle$. Por outro lado, dado $f^{i} \in\langle f\rangle$ temos

$$
\alpha^{f^{i}}=\alpha\left[\alpha, f^{i}\right]=\alpha\left(f^{i}\right)^{2}=\alpha\left(f^{2}\right)^{i}
$$

Portanto, $\alpha$ e $\beta$ conjugados se, e somente se, existe um inteiro $i$ tal que $\alpha\left(f^{2}\right)^{i}=\beta=$ $\alpha f$. Isso significa que $f$ é alguma potência de $f^{2}$, ou seja, significa que $f^{2}$ gera o subgrupo $\langle f\rangle$. Por outro lado, sabemos que $f^{2}$ gera $\langle f\rangle$ se, e somente se, $|\langle f\rangle|$ é ímpar, o que conclui o teorema.

\section{Automorfismos cindidos}

A fim de entender melhor a classe de grupos metacíclicos que consideramos neste trabalho precisamos da seguinte definição.

Definição 1.7.3. Um automorfismo $\alpha$ de um grupo $F$ é dito automorfismo cindido de ordem $p$ se

$$
\alpha^{p}=1 \quad e \quad x \cdot x^{\alpha} \cdot x^{\alpha^{2}} \cdots \cdot x^{\alpha^{p-1}}=1,
$$

para todo $x \in F$.

Note que não excluímos o caso onde $\alpha=1$, o qual implica que $F$ possui expoente $p$. Outro exemplo é fornecido se $F$ é abeliano e $\alpha$ age sobre $F$ levando cada elemento em seu inverso, neste caso $\alpha$ é um automorfismo de ordem 2. Em particular, se $F$ é cíclico então o produto semidireto $F\langle\alpha\rangle$ é um grupo diedral. Note ainda, que se um automorfismo $\varphi$ de ordem $p$ age livre de pontos fixos sobre $F$, como por exemplo um elemento de ordem $p$ 
do complemento de um grupo Frobenius age sobre o núcleo, então pelo item $(i i)$ do Lema 1.4.4, o automorfismo $\varphi$ é cindido.

Claramente vemos que se $\alpha$ é um automorfismo cindido de ordem $p$, então o subgrupo dos pontos fixos de $\alpha$ possui expoente $p$. Consequentemente, se $F$ é um $p^{\prime}$-grupo, então $\alpha$ é um automorfismo de $F$ livre de pontos fixos de $F$. Mais informações sobre automorfismos splitting de ordem prima podem ser encontradas em [27, Cap. 07].

Agora, seja $M=F H$ um grupo finito o qual é um produto de um subgrupo normal $F$ e um subgrupo $H$. Assuma que todos elementos em $M \backslash F$ possuem ordem prima $p$. Então, usando a identidade

$$
x \cdot x^{\alpha} \cdot x^{\alpha^{2}} \cdots \cdots x^{\alpha^{p-1}}=\left(x \alpha^{-1}\right)^{p} \alpha^{p}
$$

concluímos que um elemento não trivial $\alpha \in H$ é um automorfismo splitting de ordem $p$ de $F$, pois ambos $x \alpha^{-1}$ e $\alpha$ não pertencem a $F$.

Encerramos esta breve introdução sobre grupos metacíclicos provando alguns resultados que nos permitem entender melhor a família de grupos metacíclicos considerados neste trabalho. Sendo assim, no restante desta seção consideramos $M=F H$ um grupo finito o qual é um produto de dois subgrupos cíclicos $F$ e $H$, onde $F$ é um subgrupo normal e todos os elementos de $M \backslash F$ possuem ordem prima $p$.

Lema 1.7.4. Se o subgrupo $C_{F}(H)$ não é trivial, então ele é o subgrupo de ordem $p$ de $F$.

Demonstração. Como um elemento não trivial $\alpha \in H$ é um automorfismo splitting de ordem prima $p$ de $F$, temos que o subgrupo $C_{F}(H)$ possui expoente $p$. Por outro lado, um p-grupo finito não age sem pontos fixos sobre outro p-grupo, pelo Lema 1.4.1. Portanto, $C_{F}(H)$ é trivial se, e somente se, $F$ é um $p^{\prime}$-grupo e, caso contrário, $C_{F}(H)$ é o subgrupo de ordem $p$ de $F$.

Teorema 1.7.5. Se p é ímpar, então $F / C_{F}(H)$ é um $p^{\prime}$-grupo.

Demonstração. A fim de provar que $F / C_{F}(H)$ é um $p^{\prime}$-grupo é suficiente provar que $F / C_{F}(\alpha)$ é um $p^{\prime}$-grupo para qualquer $\alpha \in H$.

Suponha que $F / C_{F}(\alpha)$ não seja um $p^{\prime}$-grupo. Pelo Lema 1.4.1, temos que $C_{F / C_{F}(\alpha)}(\alpha)$ é não trivial, pois o $p$-subgrupo de Sylow de $F / C_{F}(\alpha)$ é $H$-invariante.

Agora, seja $\bar{x}$ um elemento não trivial de $C_{F / C_{F}(\alpha)}(\alpha)$ e seja $x$ uma imagem inversa de $\bar{x}$ em $F$. Assim, existe um elemento $b \in C_{F}(\alpha)$ tal que $x^{\alpha}=x b$, mais ainda $x^{\alpha^{i}}=x b^{i}$. 
Logo temos

$$
1=x x^{\alpha} \cdots \cdots x^{\alpha^{p-1}}=x(x b)\left(x b^{2}\right) \cdots\left(x b^{p-1}\right)=x b^{\frac{p(p-1)}{2}}=x^{p}
$$

que é uma contradição, pois todos os elementos de ordem $p$ pertencem a $C_{F}(H)$.

Note que um grupo diedral de ordem $2^{n}$, com $n \geq 3$, é um contra-exemplo para o caso $p=2$.

Corolário 1.7.6. Se p é ímpar, então $F=C_{F}(H) F_{p^{\prime}}$, onde $F_{p^{\prime}}$ é o $p^{\prime}$-subgrupo maximal de F. Além disso, se $F_{p^{\prime}}$ é não trivial, então $F_{p^{\prime}} H$ é um grupo de Frobenius com núcleo $F_{p^{\prime}}$ e complemento $H$.

\section{8 Álgebras de Lie}

Apresentamos agora algumas propriedades básicas sobre álgebras de Lie. Além disso, no final da seção apresentamos alguns resultados sobre álgebras de Lie admitindo um automorfismo sem pontos fixos, os quais usaremos no último capítulo.

Definição 1.8.1. Um espaço vetorial $L$ sobre um corpo $\mathbb{F}$, com uma operação produto $L \times L \longrightarrow L$, onde o par $(x, y)$ é levado em $[x, y]$, é chamado uma álgebra de Lie sobre $\mathbb{F}$ se os seguintes axiomas são satisfeitos:

(i) A operação produto é bilinear, isto é, para todos $x, x_{1}, x_{2}, y, y_{1}, y_{2} \in L$ e $\alpha \in \mathbb{F}$,

$$
\begin{aligned}
& {\left[x_{1}+x_{2}, y\right]=\left[x_{1}, y\right]+\left[x_{2}, y\right],} \\
& {\left[x, y_{1}+y_{2}\right]=\left[x, y_{1}\right]+\left[x, y_{2}\right],} \\
& \alpha[x, y]=[\alpha x, y]=[x, \alpha y] ;
\end{aligned}
$$

(ii) $[x, x]=0$, para todo $x \in L$;

(iii) $[x,[y, z]]+[y,[z, x]]+[z,[x, y]]=0$, para todos $x, y, z \in L$.

O axioma (iii) é conhecido como Identidade de Jacobi e o produto [a,b] é usualmente chamado de comutador de $a$ e $b$.

Dada uma álgebra lie $L$, dizemos que um subconjunto $B$ de $L$ é uma subálgebra de $L i e$, se $B$ é um subespaço vetorial de $A$ e a operação produto é fechada em $B$. Em outras palavras, se $B$ é uma álgebra de Lie contida em $L$. Dizemos que uma subálgebra $I$ é um ideal de $L$ se, dado $b \in I$, temos $[a, b] \in I$, para todos $a \in L$. 
De maneira análoga ao que foi feito para grupos, vamos definir, por indução, o comutador de $r$ elementos $x_{1}, x_{2}, \ldots, x_{r}$ como $\left[x_{1}, x_{2}, \ldots, x_{r}\right]=\left[\left[x_{1}, \ldots, x_{r-1}\right], x_{r}\right]$.

Dado um elemento $a \in L$, definimos o centralizador de $a$ em $L$ como sendo a subálgebra

$$
C_{L}(a)=\{x \in L:[x, a]=0\} .
$$

Analogamente, dado um subconjunto $H$ de $L$, definimos

$$
C_{L}(H)=\{x \in L:[x, h]=0, \text { para todo } h \in H\} .
$$

Dados dois ideais $H$ e $K$ de uma álgebra de Lie $L$, conseguimos um novo ideal $[H, K]$ de $L$, definindo $[H, K]$ como o subgrupo aditivo

$$
\langle[h, k]: h \in H \text { e } k \in K\rangle .
$$

Dado um elemento $x \in L$, definimos o operador adjunto associado a $x$ como a aplicação linear $a d x: L \longrightarrow L$ onde $a d x(y)=[y, x]$.

Definição 1.8.2. Um elemento $x$ de uma álgebra de Lie L é chamado ad-nilpotente se existe um inteiro positivo $k$ tal que $(a d x)^{k}=0$, ou seja,

$$
[y, \underbrace{x, \ldots, x}_{k}]=0, \quad \text { para todo } y \in L .
$$

Se $k$ é o menor inteiro com esta propriedade, então dizemos que $x$ é ad-nilpotente de indice $k$.

Definimos a seguir duas séries de ideais de uma álgebra de Lie $L$, que são análogas às séries derivada e central inferior de um grupo $G$.

Definição 1.8.3. A série derivada de uma álgebra de Lie L é definida como:

$$
\begin{aligned}
L^{(0)} & =L ; \\
L^{(1)} & =[L, L] ; \\
& \vdots \\
L^{(n)} & =\left[L^{(n-1)}, L^{(n-1)}\right] .
\end{aligned}
$$

Uma álgebra de Lie é dita solúvel de comprimento derivado n quando $n$ é o menor número natural tal que $L^{(n)}=0$.

Definição 1.8.4. Definimos a série central inferior de uma álgebra de Lie L do seguinte modo:

$$
\begin{aligned}
\gamma_{1}(L) & =L \\
\gamma_{2}(L) & =[L, L] ; \\
& \vdots \\
\gamma_{s+1}(L) & =\left[\gamma_{s}(L), L\right] .
\end{aligned}
$$


Dizemos que uma álgebra de Lie é nilpotente de classe c quando c é o menor número natural tal que $\gamma_{c+1}(L)=0$.

Definimos o centro $Z(L)$ de uma álgebra de Lie $L$ como

$$
Z(L)=\{z \in L \mid[z, a]=0, \text { para todo } a \in L\}
$$

Enunciamos agora versões dos Teoremas 1.1.3, 1.1.5 e 1.1.6 para álgebras de Lie. As demonstrações são análogas às da Teoria de Grupos.

Teorema 1.8.5. Dada uma álgebra de Lie L, as seguintes afirmações são equivalentes:

(i) $\gamma_{c+1}(L)=0$;

(ii) $\left[x_{1}, x_{2}, \ldots, x_{c+1}\right]=0$, para todos $x_{1}, x_{2}, \ldots, x_{c+1} \in L$;

Teorema 1.8.6. Uma álgebra de Lie L é nilpotente de classe $c>1$ se, e somente se, $L / Z(L)$ é nilpotente de classe $c-1$.

Teorema 1.8.7. Se uma álgebra de Lie L é gerada por um subconjunto $X$, então $L$ é nilpotente de classe $\leq c$ se, e somente se, todo comutador de peso $c+1$ com entradas em $X$ é igual a 0 .

Teorema 1.8.8 (Hall [18]). Seja $N$ um ideal de uma álgebra de Lie L. Se $N$ é nilpotente de classe $k$ e $L /[N, N]$ é nilpotente de classe $c$, então $L$ é nilpotente e sua classe de nilpotência é limitada por $(c-1) \frac{k(k+1)}{2}+k$.

Claramente o conceito de automorfismo livre de pontos fixos, definido para grupos, pode ser interpretado para uma álgebra de Lie. Dito isso, finalizamos esta seção com dois resultados importantes sobre álgebras de Lie (ou anéis de Lie) que são análogos aos Teoremas 1.4.8 e 1.4.9, uma demonstração pode ser encontrada em [27, Cap. 4].

Teorema 1.8.9 (Kreknin). Se uma álgebra de Lie L admite um automorfismo livre de pontos fixos de ordem n, então L é solúvel de comprimento derivado no máximo $2^{n-1}-1$.

É interessante comparar o teorema acima com o Teorema 1.4.9. Para uma álgebra de Lie, admitindo um automorfismo livre de pontos fixos de ordem $n$, temos uma cota superior para o comprimento derivado. No caso onde um grupo finito admite um automorfismo livre de pontos fixos de ordem $n$, sabemos apenas que tal grupo é solúvel, não sabemos se existe uma cota superior para o comprimento derivado.

Teorema 1.8.10 (Higman, Kreknin, Kostrikin). Se uma álgebra de Lie admite um automorfismo livre de pontos fixos de ordem prima $p$, então L é nilpotente de classe $h(p)$, onde $h(p)$ é um número que depende apenas de $p$ e é no máximo $\frac{(p-1)^{2^{p-1}-1}-1}{p-2}$. 


\section{9 Álgebra de Lie associada a um $p$-grupo}

O objetivo principal aqui é apresentar uma álgebra de Lie associada a um p-grupo a qual nos permite obter subgrupos potentes de índice limitado em função de parâmetros convenientes. Vale relembrar que existem outras maneiras de associar uma álgebra ou anel de Lie a um grupo, veja por exemplo [26], [27].

Seja $p$ um número primo arbitrário e fixado. Dado um grupo $G$, fixamos

$$
D_{i}=D_{i}(G)=\prod_{j p^{k} \geq i} \gamma_{j}(G)^{p^{k}}
$$

Os subgrupos $D_{i}$ são chamados de série de Jenning-Zassenhaus do grupo G. Esta série satisfaz as inclusões

$$
\left[D_{i}, D_{j}\right] \leq D_{i+j} \text { e } D_{i}^{p} \leq D_{p i}
$$

para todos $i, j$. Estas propriedades permitem construir uma álgebra de Lie $D L(G)$ sobre $\mathbb{F}_{p}$, o corpo com $p$ elementos, da seguinte maneira: considere os quocientes

$$
L_{i}=D_{i} / D_{i+1}
$$

como espaços vetoriais sobre $\mathbb{F}_{p}$ e, defina $D L(G)$ como a soma direta destes espaços

$$
D L(G)=\bigoplus_{i} L_{i}
$$

Os espaços vetoriais $L_{i}$ são chamados de espaços homogêneos, e seus elementos de elementos homogêneos de grau $i$. O comutador em $G$ induz uma operação binária $[\cdot, \cdot]$ em $D L(G)$ da seguinte forma: para elementos homogêneos $x D_{i+1} \in D_{i} / D_{i+1}$ e $x D_{j+1} \in$ $D_{j} / D_{j+1}$ definimos o produto por

$$
\left[x D_{i+1}, y D_{j+1}\right]=[x, y] D_{i+j+1} \in D_{i+j} / D_{i+j+1}
$$

e em seguida estendemos para elementos arbitrários de $D L(G)$ por linearidade. Feito isso, o espaço $D L(G)$ com as operações + e $[\cdot, \cdot]$ se torna uma álgebra de Lie sobre $\mathbb{F}_{p}$.

A seguir apresentamos um lema devido a Lazard que relaciona a ordem dos elementos de $G$ e o índice do operador adjunto associado a elementos homogêneos de $D L(G)$. Para um elemento $x \in D_{i} \backslash D_{i+1}$ denotamos por $\bar{x}$ o elemento $x D_{i}$ de $D L(G)$.

Lema 1.9.1 (Lazard [30]). Para todo $x \in G$, temos $(a d \bar{x})^{p}=a d \overline{x^{p}}$. Consequentemente, se $x$ possui ordem $p^{r}$, então $\bar{x}$ é ad-nilpotente de índice no máximo $p^{r}$.

Como um corolário do Lema de Lazard, temos o seguinte resultado. 
Corolário 1.9.2. Suponha que G seja um p-grupo o qual é uma extensão de um grupo nilpotente de classe c por um grupo de expoente e. Então, para qualquer $x \in G, \bar{x} e ́$ ad-nilpotente de indice no máximo $(c+1) e$.

Demonstração. Claramente podemos assumir que e é uma potência de $p$. Agora, como $G^{e}$ é um subgrupo normal nilpotente de classe no máximo $c$ e $\left[y, x^{e}\right] \in G^{e}$, para quaisquer $x, y \in G$, concluímos usando o lema de Lazard que

$$
[\bar{y}, \underbrace{\bar{x}, \ldots, \bar{x}}_{(c+1) e}]=[\bar{y}, \underbrace{\overline{x^{e}}, \ldots, \overline{x^{e}}}_{c+1}]=0,
$$

como desejado.

Denotamos por $L_{p}(G)$ a subálgebra gerada por $L_{1}=D_{1} / D_{2}$. O próximo teorema é o principal resultado desta seção, ele relaciona a classe de nilpotência de $L_{p}(G)$ com o índice de subgrupos potentes de $G$. Uma demonstração pode ser encontrada em [19, Proposição 1].

Teorema 1.9.3. Suponha que $G$ seja um p-grupo gerado por d elementos tal que a álgebra de Lie associada $L_{p}(G)$ é nilpotente de classe c. Então $G$ possui um subgrupo potente característico de indice limitado apenas por $c, d$ e $p$.

O próximo teorema estabelece um critério de nilpotência para a álgebra $L_{p}(G)$, sua demonstração pode ser encontrada em [25].

Teorema 1.9.4. Suponha que G seja um p-grupo gerado por d elementos tal que a álgebra de Lie associada $L_{p}(G)$ é solúvel de comprimento derivado $k$ e que as componentes $L_{i}$ sejam geradas por elementos ad-nilpotentes de índice no máximo $r$. Então $L_{p}(G)$ é nilpotente de classe limitada apenas por $d, k$ e $r$. 


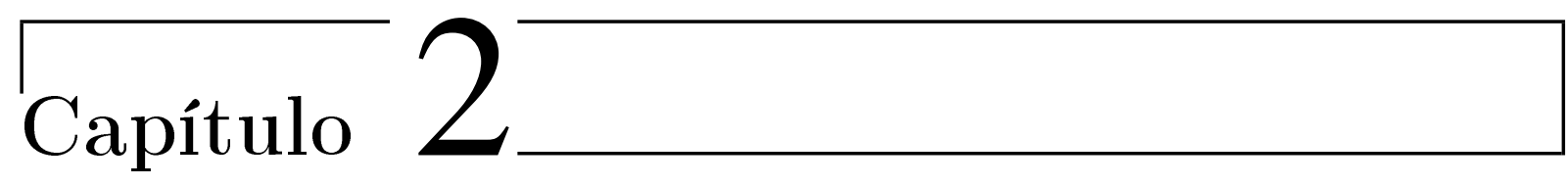

\section{Grupos metacíclicos como grupo de automorfismos}

Como mencionado na introdução, Khukhro, Makarenko e Shumyatsky provaram em [25] que se um grupo finito $G$ admite um grupo de Frobenius $K B$ como grupo de automorfismos com núcleo $K$ agindo livre de pontos fixos, então várias propriedades de $G$ estão diretamente relacionadas com as respectivas propriedades do centralizador do complemento $B$.

Em [39] Shumyatsky demonstrou resultados análogos para um grupo diedral de automorfismos. Mais especificamente ele provou que se um grupo finito $G$ admite duas involuções $\alpha$ e $\beta$ como automorfismos tal que $C_{G}(\alpha \beta)=1$, então várias propriedades de $G$ estão diretamente relacionadas às respectivas propriedades de $C_{G}(\alpha)$ e $C_{G}(\beta)$.

O objetivo desta seção é apresentar alguns resultados provados em [25] e [39], e utilizálos para iniciar nosso estudo sobre grupos metacíclicos como grupo de automorfismos. Além disso, assim como no capítulo anterior, as noções e resultados aqui apresentados servirão como ferramentas para os próximos capítulos.

\subsection{Grupos de Frobenius como grupos de automorfismos}

Aqui apresentamos alguns resultados demonstrados em [25].

Teorema 2.1.1. Seja G um grupo finito que admita um grupo de Frobenius de automorfismos $K B$ com núcleo $K$ e complemento $B$. Se $N$ é um subgrupo normal KB-invariante de $G$ tal que $C_{N}(K)=1$, então $C_{G / N}(B)=C_{G}(B) N / N$. 
É interessante observar que no teorema acima não assumimos que a ordem de $B$ é coprima com a ordem de $G$.

O próximo teorema, em um certo sentido, nos diz que é natural esperar que algumas propriedades de um grupo finito admitindo um grupo de Frobenius de automorfismos estejam relacionadas com as respectivas propriedades dos pontos fixos do complemento sempre que o núcleo age sem pontos fixos.

Teorema 2.1.2. Seja $G$ um grupo finito que admita um grupo de Frobenius de automorfismos $K B$ com núcleo $K$ e complemento $B$ tal que $C_{G}(K)=1$. Então

$$
G=\left\langle C_{G}(B)^{k} \mid k \in K\right\rangle
$$

Uma observação interessante e que será útil posteriormente é que, pelo Teorema 2.1.2 temos $G=\left\langle C_{G}(x) \mid x \in K B \backslash K\right\rangle$, ou seja, $G$ é gerado pelos centralizadores dos elementos de fora do núcleo $K$, uma vez que $C_{G}(B)^{k}=C_{G}\left(B^{k}\right)$ e $b^{k} \in K B \backslash K$, para todo $1 \neq b \in B$.

Teorema 2.1.3. Seja $G$ um grupo finito que admita um grupo de Frobenius de automorfismos $K B$ com núcleo $K$ e complemento $B$ tal que $C_{G}(K)=1$. Então

(i) $|G|=\left|C_{G}(B)\right|^{|B|}$;

(ii) o posto de $G$ é limitado por uma função que depende apenas de $|B|$ e do posto de $C_{G}(B)$;

(iii) se $C_{G}(B)$ é nilpotente, então $G$ é nilpotente.

O teorema acima, em particular o item (iii), não nos dá um limitante para a classe de nilpotência de $G$. Na verdade, em [25] foi apresentado um exemplo que mostra que, em um caso geral, a classe de nilpotência não pode ser limitada em função de $|B|$ e da classe de nilpotência de $C_{G}(B)$. No entanto, no mesmo trabalho está demonstrado o teorema seguinte, o qual é uma generalização da resposta afirmativa, dada por Makarenko e Shumyatsky, do problema 17.72(a) proposto por Mazurov no Kourovka Notebook [44].

Teorema 2.1.4. Seja KB um grupo de Frobenius com núcleo cíclico $K$ e complemento $B$ que age sobre um grupo finito $G$ de tal modo que $C_{G}(K)=1$ e $C_{G}(B)$ seja nilpotente de classe c. Então $G$ é nilpotente de classe limitada somente em função de c e $|B|$.

Embora não saibamos se no mesmo contexto do teorema acima, o expoente de $G$ pode ser limitado em função apenas de $|B|$ e do expoente do subgrupo $C_{G}(B)$, em [25] foi obtido um teorema que nos fornece informações sobre seu expoente. 
Teorema 2.1.5. Seja KB um grupo de Frobenius com núcleo cíclico $K$ e complemento $B$ que age sobre um grupo finito $G$ de tal modo que $C_{G}(K)=1$ e $C_{G}(B)$ possua expoente t. Então o expoente de $G$ é limitado somente em função de $t$ e de $|K B|$.

\subsection{Grupos diedrais como grupos de automorfismos}

A seguir, apresentamos alguns resultados provados em [39] que são os análogos, para um grupo diedral de automorfismos, dos Teoremas 2.1.4 e 2.1.5, apresentados para grupos de Frobenius de automorfismos.

Teorema 2.2.1. Seja $D=\langle\alpha, \beta\rangle$ um grupo diedral gerado por duas involuções $\alpha$ e $\beta$. Suponha que $D$ aja sobre um grupo finito $G$ de tal maneira que $C_{G}(\alpha \beta)=1$. Então $G=C_{G}(\alpha) C_{G}(\beta)$.

O teorema acima nos mostra que é natural esperar que algumas propriedades de um grupo $G$, admitindo um grupo diedral de automorfismos $D=\langle\alpha, \beta\rangle$, esteja relacionadas com as respectivas propriedades de $C_{G}(\alpha)$ e $C_{G}(\beta)$, sempre que $C_{G}(\alpha \beta)=1$.

Como uma consequência do resultado acima, Shumyatsky também provou em [39] que $|G|=\left|C_{G}(\alpha)\right|\left|C_{G}(\beta)\right|$ e que o posto de $G$ é limitado somente em termos do posto de $C_{G}(\alpha)$ e do posto de $C_{G}(\beta)$.

O próximo resultado é o análogo dos Teoremas 2.1.3(iii) e 2.1.4.

Teorema 2.2.2. Seja $D=\langle\alpha, \beta\rangle$ um grupo diedral gerado por duas involuções $\alpha$ e $\beta$. Suponha que $D$ aja sobre um grupo finito $G$ de tal maneira que $C_{G}(\alpha \beta)=1$ e ambos centralizadores $C_{G}(\alpha)$ e $C_{G}(\beta)$ são nilpotentes de classe c. Então $G$ é nilpotente e sua classe de nilpotência é limitada somente em termos de c.

O seguinte exemplo foi proposto em [39]. Ele mostra que no teorema acima não é suficiente que apenas $C_{G}(\alpha)$ seja nilpotente.

Exemplo 2.2.3. Seja $S$ um grupo abeliano elementar de ordem $13^{2}$ gerado por dois elementos a e b e seja $c$ o automorfismo de $S$ tal que $a^{c}=a^{3}$ e $b^{c}=b^{9}$. Denote por $G$ o produto semidireto $S\langle c\rangle$. O grupo $G$ admite um automorfismo $f$ de ordem 4 tal que

(1) $a^{f}=b, b^{f}=a^{-1}$ e $c^{f}=c^{-1}$.

Mais ainda, o grupo $G$ admite um automorfismo $\beta$ de ordem 2 tal que 
(2) $a^{\beta}=a^{-1}, b^{\beta}=b$ e $c^{\beta}=c$.

Seja $\alpha=\beta f$. Então $\alpha$ é um automorfismo de ordem 2. Além disso, é imediato verificar que $C_{G}(f)=1$ e $C_{G}(\alpha) \leq S$. Logo, $C_{G}(\alpha)$ é abeliano. Por outro lado, vemos que $G$ não é um grupo nilpotente.

Encerramos com o teorema análogo ao Teorema 2.1.5.

Teorema 2.2.4. Seja $D=\langle\alpha, \beta\rangle$ um grupo diedral gerado por duas involuções $\alpha$ e $\beta$. Suponha que $D$ age sobre um grupo finito $G$ de tal maneira que $C_{G}(\alpha \beta)=1$ e ambos centralizadores $C_{G}(\alpha)$ e $C_{G}(\beta)$ possuem expoente dividindo t. Então o expoente de $G$ é limitado somente em termos de $t$ e de $|D|$.

\subsection{Novos resultados}

Iniciamos agora a apresentação de novos resultados sobre grupos finitos admitindo um grupo metacíclico de automorfismos. Levando em conta que o conjunto de hipóteses usadas nos resultados do restante desta seção é sempre o mesmo, sempre que não for dito o contrário, usaremos as seguintes hipóteses e notações.

Hipóteses e Notação 2.3.1. Seja $M=F H$ um grupo finito o qual é um produto de dois subgrupos cíclicos $F$ e $H$, onde $F$ é um subgrupo normal e todos os elementos de $M \backslash F$ possuem ordem prima $p$. Seja $G$ um grupo finito admitindo $M$ como grupo de automorfismos de modo que $C_{G}(F)=1$.

Lema 2.3.2. O grupo $G$ é solúvel e sua altura de Fitting é limitada por uma função que depende apenas de $|F|$.

Demonstração. É uma consequência dos Teoremas 1.4.9 e 1.4.10, uma vez que $F=\langle f\rangle$ e $C_{G}(f)=C_{G}(F)=1$.

Lema 2.3.3. Para cada primo q divisor da ordem de $G$ existe somente um q-subgrupo de Sylow $M$-invariante em $G$.

Demonstração. Pelo Lema 1.4.6, existe um único $q$-subgrupo de Sylow $F$-invariante $P$ em $G$. Portanto, como $F$ é normal em $M$, então $P$ deve ser $M$-invariante.

Lema 2.3.4. Seja $N$ um subgrupo normal $M$-invariante de $G$. Então $C_{G / N}(F)=1$, $C_{G / N}(H)=C_{G}(H) N / N$. 
Demonstração. A igualdade $C_{G / N}(F)=1$ segue do Lema 1.4.5. Assim, precisamos provar apenas a igualdade $C_{G / N}(H)=C_{G}(H) N / N$.

Considere uma série maximal de subgrupos normais $M$-invariantes

$$
G>N=N_{1}>N_{2}>\cdots>N_{k}>N_{k+1}=1 .
$$

Os quocientes $N_{i} / N_{i+1}$ são grupos abelianos elementares. Primeiro assuma que $k=1$, ou seja, que $N$ é um $q$-subgrupo abeliano elementar para algum primo $q$. Se $p \neq q$, então o resultado segue do Lema 1.4.2. Portanto assumimos que $p=q$. Seja $F_{p^{\prime}}$ o $p^{\prime}$-subgrupo maximal de $F$. Então pelo Lema 1.4.3 temos $C_{N}\left(F_{p^{\prime}}\right)=1$ e claramente $F_{p^{\prime}} H$ é um grupo de Frobenius. Agora, usando o Teorema 2.1.1 segue que $C_{G / N}(H)=C_{G}(H) N / N$. Em outras palavras, provamos que se $N$ é um $q$-subgrupo abeliano elementar, então uma classe lateral $g N$ fixada por $H$ contém um ponto fixo de $H$.

Agora, suponha que $k>1$. Por indução sobre $k$ temos que o resultado é verdadeiro para $G / N_{k}$, ou seja, uma classe lateral $g N$ fixada por $H$ contém uma classe $x N_{k}$ fixada por $H$. Por outro lado, pelo parágrafo anterior, $x N_{k}$ possui um elemento fixado por $H$, pois $N_{k}$ é abeliano elementar. Portanto, toda classe lateral $g N$ fixada por $H$ contém um ponto fixo de $H$, como queríamos demonstrar.

Lema 2.3.5. Temos que $G=\left\langle C_{G}(x) \mid x \in M \backslash F\right\rangle$.

Demonstração. Pelo Lema 2.1.2, precisamos provar apenas para o caso em que $M$ não é um grupo de Frobenius. Neste caso, o subgrupo $A=C_{F}(H) H$ é um $p$-grupo abeliano elementar de posto 2.

Para $p=2$, o resultado segue do Teorema 2.2.1. Assim, podemos considerar $p$ um número primo ímpar.

Primeiro provamos para o caso particular em que $G$ é um $q$-grupo abeliano elementar para algum primo $q$. Suponha $p \neq q$. Então, pelo Teorema 1.6.5, $G=\left\langle C_{G}(a)\right| a \in$ $A \backslash\{1\}\rangle$. Denotamos por $b$ o elemento gerador de $C_{F}(H)$. Claramente, precisamos provar apenas que $C_{G}(b)$ é gerado por pontos fixos de elementos em $M \backslash F$. Como na prova do Lema 2.3.4, seja $F_{p^{\prime}}$ o $p^{\prime}$-subgrupo maximal de $F$. Se $F_{p^{\prime}}=1$, então pelo Corolário 1.7.6 $F=C_{F}(H)=\langle b\rangle$. Por outro lado $C_{N}(b)=C_{N}(F)=1$, por hipótese. Logo resta provar quando $F_{p^{\prime}} \neq 1$. Agora, como $C_{N}(F)=1$ e $F=\langle b\rangle F_{p^{\prime}}$ temos que $F_{p^{\prime}}$ age sem pontos fixos em $C_{N}(e)$ e $\bar{M}=F_{p^{\prime}} H$ é um grupo de Frobenius com núcleo $F_{p^{\prime}}$ e complemento $H$. Assim, $C_{N}(b)$ é gerado por elementos em $\bar{M} \backslash F_{p^{\prime}} \subset M \backslash F$ pelo Teorema 2.1.2.

Agora assuma $p=q$. Então $C_{G}\left(F_{p^{\prime}}\right)=1$ e o grupo $F_{p^{\prime}} H$ é Frobenius. Logo, usando o Teorema 2.1.2 concluímos o desejado. 
Se $G$ não é um grupo abeliano elementar, então $G$ possui um subgrupo normal abeliano elementar $N$ próprio e $M$-invariante, pois $G$ é solúvel. Argumentando por indução sobre a ordem de $G$ e usando o Lema 2.3.4 temos $N=\left\langle C_{N}(x) \mid x \in M \backslash F\right\rangle$ e $G / N=\left\langle C_{G}(x)\right| x \in$ $M \backslash F\rangle N / N$ e isto conclui a demonstração. 
Capítulo 3

\section{Altura de Fitting de grupos finitos com um grupo metacíclico de automorfismos}

Iniciamos este capítulo apresentando um resultado provado por Khukhro em [21] o qual mostra que a altura e a série de Fitting de um grupo finito com um grupo de Frobenius de automorfismos, onde o núcleo age livre de pontos fixos, está relacionada com a altura e série de Fitting do subgrupo dos pontos fixos do complemento.

Teorema 3.0.6 (Teorema 2.1 - Khukhro [21]). Suponha que um grupo finito G admita um grupo de Frobenius de automorfismos $K B$ com núcleo $K$ e complemento $B$ tal que $C_{G}(K)=1$. Então $F_{i}\left(C_{G}(B)\right)=C_{G}(B) \cap F_{i}(G)$, para todo $i$, e a altura de Fitting de $G$ é igual à altura de Fitting de $C_{G}(B)$.

Vale ressaltar que no teorema acima não assumimos que o grupo de Frobenius $K B$ seja metacíclico. No mesmo trabalho, como um corolário foi provado o seguinte resultado. Corolário 3.0.7 (Corolário 4.1 - Khukhro [21]). Suponha que um grupo finito G admita um grupo de Frobenius de automorfismos $K B$ com núcleo $K$ e complemento $B$ tal que $C_{G}(K)=1$. Então $O_{\pi}\left(C_{G}(B)\right)=O_{\pi}(G) \cap C_{G}(B)$, para todo conjunto de primos $\pi$.

Recentemente os resultados acima foram estendidos, por Ercan e Guloglu em [11] e por Collins e Flavell em [5], para o caso onde um grupo finito $G$ admite um grupo Frobeniuslike $A R$ ( $A$ é normal nilpotente e $A R / A^{\prime}$ é Frobenius) de automorfismo de tal modo que $C_{G}(A)=1$. Em ambos os casos foram colocadas certas hipóteses adicionais sobre o grupo $A R$.

O objetivo principal deste capítulo é estender os resultados acima para a família de grupos metacíclicos considerada neste trabalho. Aqui, assumimos as Hipóteses 2.3.1, que é: $M=F H$ é um grupo finito o qual é um produto de dois subgrupos cíclicos $F$ e $H$, onde 
$F$ é um subgrupo normal e todos os elementos de $M \backslash F$ possuem ordem prima $p$. Além disso, $M$ age por automorfismos sobre um grupo finito $G$ de tal modo que $C_{G}(F)=1$.

O seguinte teorema é o resultado principal deste capítulo.

Teorema 3.0.8. Suponha que $M$ aja sobre um grupo finito $G$ de tal maneira que a Hipótese 2.3.1 é satisfeita. Então $F_{i}\left(C_{G}(H)\right)=F_{i}(G) \cap C_{G}(H)$, para todo inteiro positivo $i$, e $h(G) \leq h\left(C_{G}(H)\right)+1$.

É importante relembrar que, de acordo com o Teorema 2.3.2, no teorema acima tal grupo $G$ é solúvel, uma vez que $C_{G}(F)=1$.

Observação 3.0.8.1. A igualdade $F_{i}\left(C_{G}(H)\right)=F_{i}(G) \cap C_{G}(H)$ nos diz que se $h\left(C_{G}(H)\right)=s$, então, pelo Lema 2.3.4, $H$ age sem pontos fixos em $G / F_{s}(G)$. Consequentemente $G / F_{s}(G)$ é nilpotente, pelo Teorema de Thompson [40], e temos $h(G) \leq$ $h\left(C_{G}(H)\right)+1$. Assim, o Teorema 3.0.8 estará provado uma vez estabelecida tal igualdade para todo inteiro positivo $i$.

Antes de demonstrar o Teorema 3.0.8 apresentamos dois corolários. O primeiro corolário é análogo ao Corolário 3.0.7 e o segundo é análogo aos resultados 2.1.3 e 2.2.2 apresentados no capítulo anterior.

Corolário 3.0.9. Suponha que $M$ aja sobre um grupo finito $G$ de tal maneira que a Hipótese 2.3.1 é satisfeita. Então $O_{\pi}\left(C_{G}(H)\right)=O_{\pi}(G) \cap C_{G}(H)$, para todo conjunto de primos $\pi$.

Demonstração. A inclusão $O_{\pi}\left(C_{G}(H)\right) \geq O_{\pi}(G) \cap C_{G}(H)$ é imediata. Provamos a outra inclusão argumentando por contradição. Suponha $O_{\pi}\left(C_{G}(H)\right) \not \leq O_{\pi}(G) \cap C_{G}(H)$ e considere $\bar{G}=G / O_{\pi}(G)$. Então existe um primo $q \in \pi$ tal que $O_{q}\left(C_{\bar{G}}(H)\right) \neq 1$. Temos $O_{q}\left(C_{\bar{G}}(H)\right) \leq F\left(C_{\bar{G}}(H)\right)=F(\bar{G}) \cap C_{\bar{G}}(H)$ pelo Teorema 3.0.8, assim $O_{q}(\bar{G}) \neq 1$, o que é impossível pois $O_{\pi}(\bar{G})=1$.

Corolário 3.0.10. Suponha que $M$ aja sobre um grupo finito $G$ de tal maneira que $C_{G}(F)=1$ e $C_{G}(a)$ é nilpotente, para todos a $\in M \backslash F$. Então $G$ é nilpotente.

Demonstração. Pelo Lema 2.3.5, $G=\left\langle C_{G}(x) \mid x \in M \backslash F\right\rangle$. Logo é suficiente provar que $C_{G}(x) \subset F(G)$, para todo $x \in M \backslash F$.

Seja $x \in M \backslash F$ e considere $H=\langle x\rangle$. Assim, $M=F H$ e, por hipótese $C_{G}(x)=$ $C_{G}(H)$ é nilpotente. Logo pelo Teorema 3.0.8, temos $C_{G}(x) \subset F(G)$, como queríamos demonstrar. 
Vale observar que, para o caso onde $M$ é um grupo de Frobenius com núcleo $F$ e complemento $H$, o resultado acima segue do Teorema 2.1.3. Além disso, se $p=2$, então $|H|=2$ e, para $1 \neq \alpha \in H$ e $f \in F$, temos

$$
1=(f \alpha)^{2}=f f^{\alpha},
$$

ou seja, $f^{\alpha}=f^{-1}$ e $M$ é um grupo diedral gerado por $\alpha$ e $\beta=\alpha f$. Portanto, para $p=2$ o resultado acima segue do Teorema 2.2.2.

O exemplo a seguir mostra que para um caso mais geral, onde um grupo finito $G$ admite um grupo metacíclico de automorfismos $M=F H$ tal que $C_{G}(F)=1$ e nem todos os elementos de $M \backslash F$ possuem ordem $p$, podemos ter $F_{i}\left(C_{G}(H)\right) \not \leq F_{i}(G) \cap C_{G}(H)$.

Exemplo 3.0.11. Seja $S$ um grupo abeliano elementar de ordem $13^{3}$ gerado por $v_{1}, v_{2} e$ $v_{3}$. Seja K um grupo de automorfismos de $S$ gerado por a e $b$ tal que:

(1) $v_{1}^{a}=v_{1}, v_{2}^{a}=v_{2}^{-1}$ e $v_{3}^{a}=v_{3}^{-1}$.

(2) $v_{1}^{b}=v_{1}^{-1}, v_{2}^{b}=v_{2}$ e $v_{3}^{b}=v_{3}^{-1}$.

O grupo K é um grupo abeliano elementar de ordem 4. Denote por $G$ o produto semidireto SK. O grupo $G$ admite um automorfismo $f$ sem pontos fixos de ordem 9 tal que:

(3) $v_{1}^{f}=v_{2}, v_{2}^{f}=v_{3}$ e $v_{3}^{f}=v_{1}^{3}$.

(4) $a^{f}=b, b^{f}=a b$.

Mais ainda, o grupo $G$ admite um automorfismo $\alpha$ de ordem 3 tal que:

(5) $v_{1}^{\alpha}=v_{1}, v_{2}^{\alpha}=v_{2}^{3}$ e $v_{3}^{\alpha}=v_{3}^{9}$.

(6) $a^{\alpha}=a$ e $b^{\alpha}=b$.

Assim, $f^{\alpha}=f^{4}$ (o que implica que $f \alpha^{-1}$ possui ordem 9). Por outro lado, $F\left(C_{G}(\alpha)\right)=$ $\left\langle v_{1}, a\right\rangle$ e $F(G)=S$, ou seja, $F\left(C_{G}(\alpha)\right) \not \leq F(G)$.

\subsection{Um resultado auxiliar sobre $\mathbb{F} G$-módulos}

Aqui utilizamos essencialmente as ferramentas e resultados desenvolvidos na Seção 1.6. Tais resultados nos permitem demonstrar a proposição seguinte, a qual será a chave para a demonstração do Teorema 3.0.8. A grosso modo, o que provamos aqui é que se $F(G)$ é um espaço vetorial (abeliano elementar), então os elementos que agem trivialmente sobre $C_{F(G)}(H)$ também agem trivialmente sobre todo o subgrupo $F(G)$. 
Definição 3.1.1. Sejam $G$ um grupo, $k$ um corpo e $W$ um $k G$-módulo. O núcleo da representação de $G$ sobre $W$ é o subgrupo:

$$
\operatorname{Ker}(G \text { sobre } W)=\left\{g \in G \mid w^{g}=w, \text { para todo } w \in W\right\}
$$

Proposição 3.1.2. Seja $M=F H$ um grupo finito o qual é um produto de dois subgrupos cíclicos $F$ e $H$, onde $F$ é um subgrupo normal e todos os elementos de $M \backslash F$ possuem ordem prima p. Seja $Q M$ um grupo finito onde $Q$ é q-subgrupo normal tal que $C_{Q}(F)=1$. Suponha que $W$ seja um $k Q M$-módulo irredutivel onde $k$ é um corpo com característica $r \neq q$ e, além disso, suponha $C_{W}(F)=0$. Então temos

$$
\operatorname{Ker}\left(C_{Q}(H) \text { sobre } C_{W}(H)\right)=\operatorname{Ker}\left(C_{Q}(H) \text { sobre } W\right) \text {. }
$$

Provaremos a proposição por contradição. Suponha que a proposição seja falsa e escolha um contraexemplo com menor número $\operatorname{dim}_{k} W+|Q M|$.

Lema 3.1.3. Podemos assumir que o corpo $k$ é um corpo de decomposição, para todos os subgrupos de $Q M$.

Demonstração. Estendemos o corpo $k$ para um corpo finito $\bar{k}$ o qual é um corpo de decomposição para $Q M$, ou seja, os autovalores dos elementos de $Q M$ (visto como transformações lineares) pertencem a $\bar{k}$. Agora obtemos um $\bar{k} Q M$-módulo $\widetilde{W}=W \otimes_{k} \bar{k}$. Como consequência, temos $\operatorname{dim}_{k} W=\operatorname{dim}_{\bar{k}} \widetilde{W}$ e $C_{\widetilde{W}}(H)=C_{W}(H) \otimes_{k} \bar{k}$. Portanto, usando o Teorema de Noether e Deuring 1.6.8, podemos provar o teorema para $\widetilde{W}$ no lugar de $W$.

Lema 3.1.4. $Q$ age fielmente sobre $W$.

Demonstração. Assuma que $K=\operatorname{Ker}(Q$ sobre $W) \neq 1$ e seja $\bar{Q}=Q / K$. Considere a ação do grupo $\bar{Q} M$ sobre $W$. Por minimalidade, temos

$$
\operatorname{Ker}\left(C_{\bar{Q}}(H) \text { sobre } C_{W}(H)\right)=\operatorname{Ker}\left(C_{\bar{Q}}(H) \text { sobre } W\right) \text {. }
$$

Isto é uma contradição, pois $C_{\bar{Q}}(H)=\overline{C_{Q}(H)}$ pelo Lema 2.3.4. Assim, podemos assumir que $Q$ age fielmente sobre $W$.

Pelo Teorema de Clifford 1.6.6, podemos decompor $W$ em uma soma direta de componentes de Wedderburn $W_{i}$ em relação a $Q$

$$
W=W_{1} \oplus \cdots \oplus W_{t}
$$

Relembramos que o subgrupo $M$ age transitivamente sobre o conjunto $\left\{W_{1}, \ldots, W_{t}\right\}$. 
Agora, seja $c \in \operatorname{Ker}\left(C_{Q}(H)\right.$ sobre $\left.C_{W}(H)\right)$. Pelo Lema 3.1.4 podemos escolher uma componente de Wedderburn $W_{1}$ tal que $c$ age não trivialmente. Nossa contradição decorrerá deste fato.

Notação 3.1.5. No restante deste capítulo, $\Omega$ denotará o conjunto das componentes de Wedderburn $\left\{W_{1}, \ldots, W_{t}\right\}$ e $W_{1}$ denotará uma componente sobre a qual c age não trivialmente. Também assumiremos $H=\langle\alpha\rangle$.

Lema 3.1.6. O elemento c age trivialmente sobre as componentes de qualquer H-órbita regular em $\Omega$.

Demonstração. Considere a $H$-órbita regular $\left\{W_{j}, W_{j}^{\alpha}, \ldots, W_{j}^{\alpha^{p-1}}\right\}$. O elemento $c$ deixa invariante todas as componentes $W_{j}^{\alpha^{i}}$, pois $c \in Q$. Por outro lado, $c$ age trivialmente sobre todos elementos da forma $w_{j}+w_{j}^{\alpha}+\cdots w_{j}^{\alpha^{p-1}} \in C_{W}(\alpha)$. Portanto, $c$ deve agir trivialmente sobre todas as componentes da $H$-órbita.

Pelo Lema 3.1.6 temos $W_{1}^{\alpha}=W_{1}$, em outras palavras, $W_{1}$ é uma $H$-órbita não regular. Note que se $H$ age trivialmente sobre $W_{i}$, então $W_{i} \leq C_{W}(H)$ e, consequentemente, $c$ também age trivialmente sobre $W_{i}$. Assim, $H$ age não trivialmente sobre $W_{1}$.

Denotaremos por $C_{Q}\left(W_{i}\right)$ o núcleo da ação de $Q$ sobre a componente de Wedderburn $W_{i}$, ou seja, $C_{Q}\left(W_{i}\right)=\left\{x \in Q \mid v^{x}=v\right.$, para todo $\left.v \in W_{i}\right\}$.

Seja $E$ o estabilizador de $W_{1}$ em $F$, assim o estabilizador de $W_{1}$ em $M$ é $E H$. Em particular, o grupo $E H$ age por automorfismos sobre $Q / C_{Q}\left(W_{1}\right)$. Além disso, como $F$ é abeliano, o subgrupo $E$ é estabilizador de todas componentes $W_{i}$.

Como $C_{W}(F)=0$, temos que a soma $\sum_{f \in F} v^{f}=0$, para todo $v \in W_{1}$. Assim, como a soma das componentes é direta, concluímos que $E$ deve ser não trivial. Além disso, como $W_{1}^{\alpha}=W_{1}$, vemos que $F$ age transitivamente sobre $\Omega$ e induz uma ação de $F / E$, logo temos $W=W_{1}^{\bar{F}}$.

Obviamente o elemento $\alpha \in H$ age sobre $F / E$ e assim podemos descrever a ação de $H$ sobre o conjunto de componentes $\Omega=\left\{W_{1}^{\bar{f}} \mid \bar{f} \in \bar{F}\right\}$ do seguinte modo:

$$
\left(W_{1}^{\bar{f}}\right)^{\alpha}=W_{1}^{\bar{f} \alpha}=W_{1}^{\alpha \alpha^{-1} \bar{f} \alpha}=W_{1}^{\bar{f}^{\alpha}},
$$

onde $\bar{f} \in F / E$. Em particular, quando o grupo $F / E$ não é um $p^{\prime}$-grupo, temos que os elementos de ordem $p$ em $F / E$ são os únicos que comutam com $H$ e daí temos o seguinte lema.

Lema 3.1.7. O subgrupo $H$ possui exatamente uma órbita não regular em $\Omega$ quando $F / E$ é um p'-grupo (a saber $\left.\left\{W_{1}\right\}\right)$ e, caso contrário, $H$ possui exatamente $p$ órbitas não regulares (a saber $\left\{W_{1}^{a} \mid a \in C_{F / E}(H)\right\}$ ). 
Denotamos por $\bar{A}$ o subgrupo $C_{F / E}(H)$ (os pontos fixos de $H$ em $F / E$ ), o qual pode ser trivial, e denotamos por $A$ sua pré-imagem em $F$.

Os Lemas 3.1.6 e 3.1.7 nos permitem concluir que $c$ age trivialmente sobre todas as componentes de Wedderburn de $\Omega$ diferentes de $W_{1}^{a}$, onde $a \in A$. Portanto, para qualquer $f \in F \backslash A$ o elemento $c$ age trivialmente sobre $W_{1}^{a f}$, para todo $a \in A$, pois af $\notin A$. Em outras palavras, $c^{x} \in \bigcap_{a \in A} C_{Q}\left(W_{1}^{a}\right)$ para qualquer $x \in F \backslash A$. Em particular, temos

$$
c^{y x} \in \bigcap_{a \in A} C_{Q}\left(W_{1}^{a}\right) \text {, para qualquer } y \in A \text { e } x \in F \backslash A,
$$

pois neste caso $y x \in F \backslash A$.

Dividimos o final da demonstração em dois casos: primeiro demonstramos para o caso $(|Q|, p)=1$ e depois para o caso $(|Q|, p)=p$.

\section{Caso 1}

Aqui assumimos $(|Q|, p)=1$.

Proposição 3.1.8. O subgrupo $Q$ é abeliano.

Demonstração. É fácil checar a igualdade $C_{Q}\left(W_{1}^{g}\right)=\left(C_{Q}\left(W_{1}\right)\right)^{g}$, para qualquer $g \in F$. Fixe o subgrupo $K=\bigcap_{a \in A} C_{Q}\left(W_{1}^{a}\right)=\bigcap_{a \in A}\left(C_{Q}\left(W_{1}\right)\right)^{a}$, o qual é $A$-invariante.

Denotaremos $Q / K$ por $\bar{Q}$ e usaremos uma barra superior para denotar seus elementos. Seja $\zeta_{2}(\bar{Q})$ o segundo centro de $\bar{Q}$.

Como $c \in C_{Q}(H)$, obviamente vale

$$
\left[[H, \bar{c}], \zeta_{2}(\bar{Q})\right]=\left[1, \zeta_{2}(\bar{Q})\right]=1
$$

Também temos

$$
\left[\left[\bar{c}, \zeta_{2}(\bar{Q})\right], H\right] \leq[Z(\bar{Q}), H]=1,
$$

pois, para todo $a \in A$, a componente $W_{1}^{a}$ é $H$-invariante e $Z(\bar{Q})$ é representado por uma transformação linear escalar sobre a componente homogênea $W_{1}^{a}$. Pelo Lema dos Três Subgrupos temos

$$
\left[\left[\zeta_{2}(\bar{Q}), H\right], \bar{c}\right]=1
$$

Pela escolha do elemento $c \in Z\left(C_{G}(H)\right)$, também temos

$$
\left[C_{\zeta_{2}(\bar{Q})}(H), \bar{c}\right]=1,
$$

pois $C_{Q}(H)$ cobre $C_{\bar{Q}}(H)$ de acordo com o Teorema 2.3.4. A ordem de $Q$ é coprima com $|H|$ por hipótese, logo vale

$$
\zeta_{2}(\bar{Q})=C_{\zeta_{2}(\bar{Q})}(H)\left[\zeta_{2}(\bar{Q}), H\right]
$$


Portanto, obtemos de (3.2) e (3.3)

$$
\left[\bar{c}, \zeta_{2}(\bar{Q})\right]=1
$$

Agora, como $Q=\left\langle c^{F}\right\rangle$, usando (3.1) obtemos $\bar{Q}=\left\langle\bar{c}^{A}\right\rangle$. Levando em conta que $\bar{Q}$ é $A$-invariante, podemos aplicar conjugação por $g \in A$ em (3.3) para obter

$$
\left[\bar{c}^{g}, \zeta_{2}(\bar{Q})^{g}\right]=\left[\bar{c}^{g}, \zeta_{2}(\bar{Q})\right]=1
$$

Como resultado,

$$
\left[\left\langle\bar{c}^{A}\right\rangle, \zeta_{2}(\bar{Q})^{g}\right]=\left[\bar{Q}, \zeta_{2}(\bar{Q})\right]=1 .
$$

Isso significa que $\zeta_{2}(\bar{Q}) \leq Z(\bar{Q})$, ou seja, $\bar{Q}$ é abeliano e consequentemente $Q^{\prime} \leq$ $\bigcap_{a \in A}\left(C_{Q}\left(W_{1}\right)\right)^{a}$. Por outro lado, como $F$ age transitivamente sobre $\Omega$, devemos ter

$$
Q^{\prime} \leq \bigcap_{x \in F}\left(C_{Q}\left(W_{1}\right)\right)^{x}=\operatorname{Ker}(Q \text { sobre } W)=1
$$

Portanto, $Q$ é abeliano.

Lema 3.1.9. Temos $C_{F}(H) \leq E$, onde $E$ denota o estabilizador de $W_{1}$ em $F$.

Demonstração. Lembre-se que $c$ age não trivialmente sobre $W_{1}$. Sabemos pela Proposição 3.1.8 e Lema 1.6.1 que $c$ age como uma transformação escalar sobre $W_{1}$ e, por hipótese, $c$ age trivialmente sobre $C_{W_{1}}(H)$, assim $W_{1}$ não possui pontos fixos não triviais de $H$.

Seja $r$ a característica do corpo $k$. Relembramos que $E$ é não trivial e $E=E_{r} \times E_{r^{\prime}}$, onde $E_{r}$ é o $r$-subgrupo de Sylow de $E$ e $E_{r^{\prime}}$ é seu complemento. Agora, usando o Lema 1.4.3 obtemos $C_{W}\left(F_{r^{\prime}}\right)=1$, onde $F_{r^{\prime}}$ é o $r^{\prime}$-subgrupo maximal de $F$. Assim, temos $\sum_{g \in F_{r^{\prime}}} v^{g}=0$ para $v \in W_{1}$. Consequentemente, o subgrupo $E_{r^{\prime}}$ deve ser não trivial.

A componente $W_{1}$ é um $k E_{r^{\prime}}$-módulo completamente irredutível, pois $\left(\left|E_{r^{\prime}}\right|, r\right)=1$. Assim,

$$
W_{1}=\bigoplus_{i=1}^{l} U_{i}
$$

para $k E_{r^{\prime}}$-submódulos irredutíveis $U_{i}$. Claramente $E_{r^{\prime}}$ é representado por uma transformação linear escalar na ação sobre $U_{i}$. Além disso, cada componente $U_{i}$ deve ser $H$-invariante, pois caso contrário a órbita de um elemento não trivial de $U_{i}$ seria um ponto fixo de $H$. Portanto, $y^{\alpha}=y$ para todo $y \in E_{r^{\prime}}, \alpha \in H$ e consequentemente temos $E_{r^{\prime}}=C_{F}(H)$, pois $C_{F}(H)$ possui ordem prima.

Lema 3.1.10. O elemento c age trivialmente sobre todas componentes de Wedderburn $W_{i}$ diferentes de $W_{1}$. 
Demonstração. Como denotado antes, $E$ é o estabilizador de $W_{1}$ em $F$. Se $p$ é ímpar, então $F / E$ é um $p^{\prime}$-grupo, pelos Teorema 1.7.5 e Lema 3.1.9. Assim, pelo Lema 3.1.7, o resultado é verdadeiro para um primo $p$ ímpar.

Agora, suponha que $p=2$. Pelo Lema 3.1.7, precisamos apenas mostrar que $c$ age trivialmente sobre $W_{1}^{a}$, com a é um ponto fixo de $H$ em $F / E$.

Relembramos que pela Proposição 3.1.8 e pelo Lema 1.6.1, c age como uma transformação escalar sobre $W_{1}$ e, por hipótese, $c$ age trivialmente sobre $C_{W_{1}}(\alpha)$. Assim $W_{1}$ não possui pontos fixos de $\alpha$. Consequentemente a característica do corpo é diferente de 2 e $v^{\alpha}=-v$, para todo $v \in W_{1}$. Diante disso, temos que dado $e \in E$ vale

$$
-v^{e}=\left(v^{e}\right)^{\alpha}=\left(v^{\alpha}\right)^{e^{-1}}=-v^{e^{-1}}
$$

com $v \in W_{1}$. Como resultado, $e$ é o elemento de ordem 2 em $F$ e assim $E=C_{F}(\alpha)$.

Diante disso, concluímos que $\alpha$ e $e$ são involuções e ambas agem livre de pontos fixos sobre $W_{1}$. Em outras palavras, $x^{\alpha}=-x$ e $x^{e}=-x$, para todo $x \in W_{1}$. Agora, para completar a prova, precisamos apenas provar que $W_{1}^{a}$ possui um ponto fixo não trivial de $\alpha$. Note que $a^{\alpha}=a^{-1}=a e$, pois $a$ é o ponto fixo de $\alpha$ em $F / E$. Temos

$$
\left(x^{a}\right)^{\alpha}=\left(x^{\alpha}\right)^{a^{-1}}=\left(x^{\alpha}\right)^{e a}=(-x)^{e a}=x^{a} .
$$

Portanto, $x^{a} \in W_{1}^{a}$ é um ponto fixo de $\alpha$ como desejado.

Agora, usando (3.1) e o Lema 3.1.10, concluímos que $c^{x}$ age trivialmente sobre $W_{1}$ qualquer que seja $x \in F \backslash E$.

Podemos assumir que $C_{Q}\left(F_{q^{\prime}}\right)=1$ pelo Lema 1.4.3, onde $F_{q^{\prime}}$ é o $q^{\prime}$-subgrupo maximal de $F$. Para simplificar a notação fixamos $X=F_{q^{\prime}}$.

O produto $\prod_{x \in X} c^{x}$ claramente é um ponto fixo de $X$. Assim temos

$$
1=\prod_{x \in X} c^{x}=\prod_{y \in X \cap E} c^{y} \cdot \prod_{x \in X \backslash E} c^{x} .
$$

No segundo produto da direita, cada elemento $c^{x}$ pertence a $C_{Q}\left(W_{1}\right)$, como mencionado antes. No primeiro produto da direita de (3.5) temos $c^{y} \equiv c\left(\bmod C_{Q}\left(W_{1}\right)\right)$, para qualquer $y \in X \cap E$, pois $c$ é representado por uma transformação linear escalar na ação sobre a componente homogênea $W_{1}$.

Como resultado, passando para congruência módulo $C_{Q}\left(W_{1}\right)$ em (3.5), obtemos

$$
1 \equiv \prod_{y \in X \cap E} c^{y} \equiv c^{|X \cap E|}\left(\bmod C_{Q}\left(W_{1}\right)\right)
$$


Portanto, $c \in C_{Q}\left(W_{1}\right)$, uma vez que $|X \cap E|$ é coprimo com $q$. Mas isto é uma contradição com a escolha de $W_{1}$ e, consequentemente, com o Lema 3.1.4.

\section{Caso 2}

Aqui supomos $(|Q|, p)=p$. Usando a mesma notação do Caso 1 , temos que $X=F_{q^{\prime}}$ possui ordem coprima com $p$ e assim o subgrupo $C_{F}(H)$ não pertence a $X$.

O subgrupo $F$ age transitivamente sobre as componentes $W_{i}$ e, portanto, sobre o conjunto de núcleos $C_{Q}\left(W_{i}\right)$. Portanto, levando em conta que $\bigcap_{i} C_{Q}\left(W_{i}\right)=1$, obtemos que a classe de nilpotência de $Q / C_{Q}\left(W_{1}\right)$ é igual à classe de nilpotência de $Q$.

Como no caso anterior, denotamos por $E$ o estabilizador de $W_{1}$ em $F$ e por $\bar{A}$ e $A$ os pontos fixos de $H$ em $F / E$ e sua imagem inversa, respectivamente.

Relembre-se que $Q / C_{Q}\left(W_{1}\right)$ é não trivial, pois $c \notin C_{Q}\left(W_{1}\right)$. Escolhemos um comutador simples $z=\left[c^{y_{1}}, \ldots, c^{y_{n}}\right]$ de peso maximal nos elementos $c^{y}, y \in A$, que não pertencem a $C_{Q}\left(W_{1}\right)$. Como $Q=\left\langle c^{A}\right\rangle C_{Q}\left(W_{1}\right)$ por (3.1), o peso deste comutador é igual à classe de nilpotência de $Q / C_{Q}\left(W_{1}\right)$, a qual é igual à classe de nilpotência de $Q$. Portanto, z pertence ao centro de $Q$. (Note que não excluímos o caso de $Q / C_{Q}\left(W_{1}\right)$ ser abeliano, onde simplesmente tomamos $z=c$.)

Como o elemento $z$ é central, o produto $\prod_{x \in X} z^{x}$ claramente é um ponto fixo de $X$ em $Q$ e, portanto, deve ser trivial. Assim, temos

$$
1=\prod_{x \in X} z^{x}=\prod_{y \in X \cap E} z^{y} \cdot \prod_{x \in X \backslash E} z^{x} .
$$

No segundo produto da direita, cada elemento $z^{x}=\left[c^{y_{1} x}, \ldots, c^{y_{n} x}\right]$ pertence a $C_{Q}\left(W_{1}\right)$, pois $x \notin A$ (já que $A \cap X=E \cap X)$ e $c^{y_{i} x} \in C_{Q}\left(W_{1}\right)$, para todo $i$, por (3.1). Para cada elemento no primeiro produto da direita de (3.6) temos que $z^{y} \equiv z\left(\bmod C_{Q}\left(W_{1}\right)\right)$, para qualquer $y \in E$. Na verdade, a imagem de $z$ em $Q / C_{Q}\left(W_{1}\right)$ também é central e assim é representado por uma transformação linear escalar sobre a componente homogênea $W_{1}$ : note também que $W_{1}$ e, portanto, $C_{Q}\left(W_{1}\right)$ são $E$-invariantes, assim a ação de $y \in X \cap E$ sobre a imagem de $z$ em $Q / C_{Q}\left(W_{1}\right)$ está bem definida.

Como um resultado, passando para congruência módulo $C_{Q}\left(W_{1}\right)$ em (3.6), obtemos

$$
1 \equiv \prod_{y \in X \cap E} z^{y} \equiv z^{|X \cap E|}\left(\bmod C_{Q}\left(W_{1}\right)\right)
$$

Portanto, $z \in C_{Q}\left(W_{1}\right)$, pois $|X \cap E|$ é coprimo com $q$. Mas isto é uma contradição com a escolha de $z$. Logo $Q / C_{Q}\left(W_{1}\right)$ é trivial, o que contradiz a escolha de $W_{1}$ e consequentemente o Lema 3.1.4, que encerra a demonstração da Proposição 3.1.2. 


\subsection{Prova do teorema principal}

A demonstração será feita por contradição. Sua maior parte consiste em provar que podemos tomar um contraexemplo minimal $G=V Q$, onde $V$ e $Q$ são subgrupos abelianos elementares $M$-invariantes e $C_{V}(Q)=1$. Mais ainda, podemos escolher um elemento $c \in Q$ tal que $c$ age trivialmente sobre $C_{V}(H)$ mas age não trivialmente sobre $V$. Feito isso, usaremos o resultado da seção anterior para obter a contradição desejada.

Usando o Lema 2.3.4, vemos que a imagem de $C_{G}(\alpha)$ cobre $C_{G / N}(\alpha)$, para qualquer subgrupo normal $D$-invariante $N$, assim temos

$$
F\left(C_{G}(\alpha)\right) N / N \leq F\left(C_{G / N}(\alpha)\right)
$$

Claramente também temos

$$
F\left(C_{G}(\alpha)\right) \cap N \leq F\left(C_{N}(\alpha)\right) .
$$

Estas duas inclusões nos permitem mostrar que certas hipóteses também são válidas para subgrupos ou quocientes de $G$. Temos ainda pelo Lema 2.3.4, que a hipótese $C_{G}(F)=1$ é inerente para quocientes por subgrupos $F$-invariantes e obviamente para tais subgrupos.

Nosso objetivo é provar que $F\left(C_{G}(H)\right)=F(G) \cap C_{G}(H)$ e assim para completar a prova precisamos apenas usar indução sobre a série de Fitting de $C_{G}(H)$, pois $C_{G / F_{i}(G)}(H)=$ $C_{G}(H) F_{i}(G) / F_{i}(G)$ para todo $i$ pelo Lema 2.3.4.

A inclusão $F\left(C_{G}(H)\right) \geq F(G) \cap C_{G}(H)$ é imediata. Agora, provamos a inclusão contrária. Por contradição, suponha que $F\left(C_{G}(H)\right) \not \leq F(G)$ e fixe $G$ satisfazendo esta condição e com a menor ordem possível.

Como $F(G)=\bigcap_{r} O_{r^{\prime}, r}(G)$, existe um primo $r$ tal que $F\left(C_{G}(H)\right) \not \leq O_{r^{\prime}, r}(G)$. Por (3.7), a imagem de $F\left(C_{G}(H)\right)$ em $G / O_{r^{\prime}}(G)$ está contida em $F\left(C_{G / O_{r^{\prime}}(G)}(H)\right)$. Também temos $C_{G / O_{r^{\prime}}(G)}(F)=1$, pelo Lema 2.3.4. Pela minimalidade de nosso contra-exemplo $G$, devemos ter $O_{r^{\prime}}(G)=1$ e $\operatorname{assim} F(G)=O_{r}(G)$.

Como $C_{G / F(G)}(F)=1$, pelo Lema 2.3.4, por minimalidade, temos

$$
F\left(C_{G / F(G)}(H)\right) \leq F_{2}(G) / F(G)
$$

e daí $F\left(C_{G}(H)\right) \leq F_{2}(G)$ por (3.7). Logo , por minimalidade temos $G=F_{2}(G)$ em vista de (3.8). Como $F(G)=O_{r}(G)$, o quociente $G / F(G)=F_{2}(G) / F(G)$ é um $r^{\prime}$-grupo 
nilpotente. Portanto existe um primo $q \neq r$ dividindo $\left|F\left(C_{G}(H)\right)\right|$. Seja $Q_{0}=O_{q}\left(C_{G}(H)\right)$. Como o quociente $G / O_{r}(G)$ é nilpotente, $C_{G}(H) / O_{r}\left(C_{G}(H)\right)$ também é nilpotente. Logo, o $q^{\prime}$-subgrupo de Hall de $C_{G}(H)$ centraliza $Q_{0}$. Consequentemente, $Q_{0} \cap Z\left(C_{G}(H)\right) \neq 1$. Sendo assim, podemos escolher um q-elemento $c \in Q_{0} \cap Z\left(C_{G}(H)\right)$.

Considere o subgrupo $\left\langle c^{M}\right\rangle=\left\langle c^{F}\right\rangle$, o menor subgrupo $M$-invariante contendo $c$. $\mathrm{O}$ subgrupo $N=O_{p}(G)\left\langle c^{M}\right\rangle$ também é $M$-invariante e, junto com o grupo de automorfismos $M$, também é um contraexemplo para o teorema. Portanto, por minimalidade, temos $G=O_{r}(G)\left\langle c^{M}\right\rangle$. Seja $Q$ o $q$-subgrupo de Sylow $M$-invariante de $G$, o qual existe pelo Lema 2.3.3. Como todos os $q$-elementos de $G$ estão contidos em $O_{r}(G) Q$, temos $G=O_{r}(G)\left\langle c^{M}\right\rangle=O_{r}(G) Q$. Claramente, temos $C_{G}(H)=C_{O_{r}(G)}(H) C_{Q}(H)$ e, portanto, $C_{Q}(H)$ é um $q$-subgrupo de Sylow de $C_{G}(H)$. Consequentemente, $Q_{0}=O_{q}\left(C_{G}(H)\right) \leq$ $C_{Q}(H)$, em particular, $c \in Q$ e devemos ter a igualdade

$$
Q=\left\langle c^{M}\right\rangle
$$

Como $c$ age fielmente sobre $O_{r}(G) / \Phi\left(O_{r}(G)\right)$, por minimalidade devemos ter também $\Phi\left(O_{r}(G)\right)=1$, e por isso $V=O_{r}(G)=F(G)$ é um $r$-grupo abeliano elementar, sobre o qual o quociente $G / V \cong Q$ age fielmente. Além disso, como $c$ age não trivialmente sobre o subgrupo $Q M$-invariante $[V, Q]$, por outro argumento de minimalidade, devemos ter $V=[V, Q]$ e assim $C_{V}(Q)=1$. Note também que $c$ centraliza $C_{V}(H)$ mas não age trivialmente sobre $V$. Nosso objetivo é obter uma contradição deste fato. Resumindo, temos as seguintes propriedades de $V$ :

(V1) $V$ é um $\mathbb{F}_{r} Q$-módulo fiel;

(V2) $C_{V}(Q)=0$

(V3) c age trivialmente sobre $C_{V}(\alpha)$;

(V4) $C_{V}(F)=0$.

Agora, podemos considerar $V$ como um $\mathbb{F}_{r} Q M$-módulo e tomar uma série com comprimento maximal de $\mathbb{F}_{r} Q M$-submódulos

$$
V=V_{1}>V_{2}>\cdots V_{n}>V_{n+1}=0
$$

Seja $W$ um dos fatores desta série, isto é, $W=V_{i} / V_{i+1}$ é um $\mathbb{F}_{r} Q M$-módulo não trivial e irredutível. Se $c$ age trivialmente sobre $V_{i} / V_{i+1}$, para todo $i$, então $c$ age trivialmente sobre $V$, pois a ordem de $c$ é coprima com a característica $r$ do corpo $k$ e, isto contradiz 
$(V 1)$. Por outro lado, $C_{W}(F)=0$, pois podemos considerar $V$ como um grupo finito sobre o qual $F$ age sem pontos fixos. Portanto, agora basta usar a Proposição 3.1.2 para concluir que $c$ age trivialmente sobre $W$ e assim obter a contradição desejada. 
Capítulo 4

\section{Expoente e leis positivas de grupos finitos com um grupo metacíclico de automorfismos}

Usaremos a expressão $r$-limitada para indicar que existe uma função $f=f(r)$, dependendo somente de $r$, que limita superiormente certo valor. Por exemplo, a classe de nilpotência dos grupos finitos que admitem um automorfismo livre de pontos fixos de ordem prima $p$ é $p$-limitada, pois é limitada pela função de Higman $h(p)$, a qual é menor do que $\frac{(p-1)^{2^{p-1}-1}-1}{p-2}$.

Analogamente, usaremos a notação $\left(r_{1}, \ldots, r_{k}\right)$-limitada para indicar que certo valor é limitado superiormente por uma função $f=f\left(r_{1}, \ldots, r_{k}\right)$, dependendo apenas de $r_{1}, \ldots, r_{k}$.

Conforme mencionado no Capítulo 2, Khukhro, Makarenko e Shumyatsky provaram em [25] que se um grupo finito $G$ admite um grupo de Frobenius $K B$ como grupo de automorfismos com núcleo cíclico $K$ agindo livre de pontos fixos, então o expoente de $G$ é limitado por $|K B|$ e pelo expoente do centralizador do complemento $B$. Além disso, em [39] Shumyatsky demonstrou um resultado análogo para um grupo diedral de automorfismos.

A primeira parte deste capítulo consiste em provar um resultado semelhante aos resultados mencionados no parágrafo anterior para a família de grupos metacíclicos considerada neste trabalho. A segunda parte é dedicada a estudar grupos diedrais como grupos de automorfismos. Mais precisamente, na segunda parte, demonstramos um teorema análogo ao seguinte teorema provado por Shumyatsky em [38].

Teorema 4.0.1 (Shumyatsky, 2011). Suponha que um grupo de Frobenius KB com núcleo 
cíclico $K$ e complemento $B$ aja sobre um grupo finito $G$ de tal maneira que $C_{G}(K)=1$ e $C_{G}(B)$ satisfaz uma lei positiva de grau $k$. Então $G$ satisfaz uma lei positiva cujo grau é limitado somente em termos de $k$ e $|K B|$.

Na segunda parte deste capítulo o teorema anterior será usado sempre que nosso grupo diedral for um grupo de Frobenius, ou seja, sempre que em nosso grupo diedral, gerado por duas involuções $\alpha$ e $\beta$, tivermos que $|\alpha \beta|$ é ímpar.

\subsection{Expoente de grupos finitos com um grupo metacíclico de automorfismos}

Aqui, como no capítulo anterior, $M=F H$ é um grupo finito o qual é um produto de dois subgrupos cíclicos $F$ e $H$, onde $F$ é um subgrupo normal e todos os elementos de $M \backslash F$ possuem ordem prima $p$. Suponha que $M$ aja como um grupo de automorfismos sobre um grupo finito $G$ de tal modo que $C_{G}(F)=1$. O objetivo desta seção é entender qual é a relação entre o expoente de $G$ e o expoente dos centralizadores dos elementos de $M \backslash F$. O seguinte teorema é o resultado principal desta seção.

Teorema 4.1.1. Suponha que $M$ aja sobre um grupo finito $G$ de tal maneira que $C_{G}(F)=$ 1 e o expoente de $C_{G}(x)$ divide e, para todos os elementos $x \in M \backslash F$. Então o expoente de $G$ é $(e,|M|)$-limitado.

Demonstração. Relembramos que o grupo $G$ possui altura de Fitting limitado por uma função dependendo apenas de $|F|$, de acordo com o Teorema 1.4.10. Sendo assim podemos usar indução sobre a altura de Fitting de $G$. Por outro lado, sabemos que o quociente dos termos da série de Fitting são nilpotentes, logo basta demonstrar o teorema para o caso onde $G$ é um grupo nilpotente. Para isso, vemos que é suficiente considerar $G$ como um $q$-grupo onde $q$ é um primo arbitrário, uma vez que um grupo nilpotente finito é o produto direto de seus subgrupos de Sylow.

A estratégia usada para provar o teorema quando $G$ é um $q$-grupo consiste basicamente em reduzi-lo para o caso onde $G$ é um grupo potente, para isso usamos os resultados apresentados na Seção 1.9 .

Pelo Lema 2.3.5 temos $G=\left\langle C_{G}(x) \mid x \in M \backslash F\right\rangle$, sendo assim $q$ divide $e$. Logo podemos assumir que é é uma potência de $q$. Claramente, para cada $x \in G$, o subgrupo $\left\langle\left\{x^{m} \mid m \in\right.\right.$ $M\}\rangle$ é um subgrupo $M$-invariante com no máximo $|M|$ geradores. Nosso objetivo é mostrar que a ordem de $x$ é $(|M|, e)$-limitada. Portanto, sem perda de generalidade 
podemos assumir que $G=\left\langle\left\{x^{m} \mid m \in M\right\}\right\rangle$, ou seja, podemos assumir que $G$ é $|M|$ gerado e seu conjunto gerador é $M$-invariante.

Qualquer grupo de automorfismos de $G$ age naturalmente sobre os grupos quocientes $L_{i}=D_{i} / D_{i+1}$ formados pelos termos da série de Jennings-Zassenhaus de $G$. Esta ação induz uma ação por grupo de automorfismos sobre a álgebra de Lie $L=L_{q}(G)$. Pelo Lema 2.3.4, o subgrupo $F$ age livre de pontos fixos sobre todos os quocientes $L_{i}$ da série de Jennings-Zassenhaus e também temos $C_{L_{i}}(H)=C_{D_{i}}(H) D_{i+1} / D_{i+1}$. Consequentemente temos $C_{L}(F)=0$, e

$$
C_{L}(x)=\bigoplus C_{D_{i}}(x) D_{i+1} / D_{i+1}
$$

para todo $x \in M \backslash F$.

Pelo Lema 2.3.5, cada componente $L_{i}$ é gerada pelos centralizadores $C_{L_{i}}(x)$, onde $x \in M \backslash F$. Mais ainda, o Lema 2.3.4 implica que todo elemento de $C_{L_{i}}(x)$ é a imagem de algum elemento de $C_{G}(x)$, cuja ordem divide $e$. Agora, utilizando o Lema de Lazard 1.9.1 segue que o grupo aditivo de $L_{i}$ é gerado por elementos que são ad-nilpotentes de índice no máximo e, mais ainda, usando o Teorema de Kreknin 1.8.9, vemos que $L$ é solúvel de comprimento $|M|$-limitado. Portanto pelo Teorema 1.9.4 temos que $L$ é nilpotente de classe $(e,|M|)$-limitada. A nilpotência de $L_{p}(G)$ implica que $G$ possui um subgrupo potente característico de índice $(e,|M|)$-limitado, pelo Teorema 1.9.3. Sendo assim é suficiente limitar o expoente de tal subgrupo potente, ou seja, podemos assumir que $G$ é um $p$-grupo potente. Por outro lado, pelo Lema 1.2.4(ii), o expoente de um grupo potente divide a ordem do seus de seus geradores. Portanto, como $G=\left\langle C_{G}(x) \mid x \in M \backslash F\right\rangle$, concluímos o teorema.

\subsection{Leis positivas em grupos finitos com um grupo diedral de automorfismos}

Dedicamos o restante deste capítulo para a demonstração do seguinte teorema.

Teorema 4.2.1. Seja $D=\langle\alpha, \beta\rangle$ um grupo diedral gerado por duas involuções $\alpha$ e $\beta$. Suponha que $D$ aja sobre um grupo finito $G$ de tal modo que $C_{G}(\alpha \beta)=1$ e ambos $C_{G}(\alpha)$ e $C_{G}(\beta)$ satisfazem uma lei positiva de grau $k$. Então $G$ satisfaz uma lei positiva de grau limitado somente por $k$ e $|D|$.

Aqui, assim como no teorema anterior, o grupo $G$ possui altura de Fitting limitado por uma função dependendo apenas de $|F|$, de acordo com o Teorema 1.4.10. A estratégia usada para provar o teorema consiste em demonstrá-lo primeiramente para o caso em que 
$G$ é um p-grupo, para um primo arbitrário $p$ e, em seguida, usar indução sobre altura de Fitting de $G$.

Para provar o teorema quando $G$ é um $p$-grupo, novamente reduzimos ao caso onde $G$ é um grupo potente. Para isso precisamos antes associar a $G$ a álgebra de Lie $L_{p}(G)$ definida na Seção 1.9 e mostrar que ela é nilpotente e sua classe de nilpotência é $(k,|D|)$-limitada.

Utilizaremos também o resultado de Burns, Macedońska e Medvedev [3] mencionado na Seção 1.3, o qual diz que existem funções $c(k)$ e $e(k)$ dependendo somente de $k$ tais que qualquer grupo finito satisfazendo uma lei positiva de grau $k$ é extensão de um grupo nilpotente de classe no máximo $c(k)$ por um grupo de expoente dividindo $e(k)$.

A fim de tornar a demonstração mais organizada, dividimos o restante do capítulo em algumas subseções, cada uma delas abordando assuntos diferentes que serão usados como resultados auxiliares. Por fim, usamos tais resultados e concluímos a demonstração do Teorema 4.2.1 no final do capítulo.

\section{Um critério de nilpotência para álgebras de Lie}

Ao longo desta subseção, denotamos por $L$ uma álgebra de Lie sobre um corpo de característica diferente de 2 tal que seu grupo aditivo seja finito (ou localmente finito). Se $X \subset L$ é um subconjunto de $L$, denotamos por $\langle X\rangle$ a subálgebra gerada por $X$. Um elemento de $L$ é dito homogêneo de peso $\omega$ com respeito ao conjunto gerador $X$ se ele pode ser escrito como um polinômio homogêneo de Lie de peso $\omega$ em elementos de $X$. Por exemplo, se $x_{1}, x_{2} \in X$, então $\left[x_{1}, x_{2}\right]$ é um elemento homogêneo de peso 2 . Note que os elementos homogêneos de peso 1 em elementos de $X$ são as combinações lineares de elementos em $X$. Relembramos que um elemento $x$ de uma álgebra de Lie $L$ é chamado ad-nilpotente se existe um inteiro positivo $k$ tal que $(\operatorname{ad} x)^{k}=0$, que significa,

$$
[y, \underbrace{x, \ldots, x}_{k}]=0 \text { para qualquer } y \in L .
$$

Se $k$ é o menor inteiro com esta propriedade, então dizemos que $x$ é ad-nilpotente de índice $k$. No que segue, o termo homogêneo significa sempre homogêneo com respeito a um conjunto gerador fixado $X$.

Lema 4.2.2. Seja $D=\langle\alpha, \beta\rangle$ um grupo diedral gerado por duas involuções $\alpha$ e $\beta$ que age sobre uma álgebra de Lie metabeliana $L$ de modo que $C_{L}(\alpha \beta)=0$. Seja $X \subset L$ um subconjunto D-invariante tal que $L=\langle X\rangle$ e suponha que exista um inteiro positivo $t$ para o qual todo elemento homogêneo contido em $C_{L}(\alpha)$ ou $C_{L}(\beta)$ é ad-nilpotente de índice no 
máximo $t$ em $C_{L}(\alpha)$ ou $C_{L}(\beta)$, respectivamente. Então

$$
[L, \underbrace{z, \ldots, z}_{3 t}]=0
$$

para todo elemento homogêneo $z \in L$.

Demonstração. Por simplicidade, dada uma subálgebra $K$ de $L$, denotamos por $K_{\alpha}$ e $K_{\beta}$ as subálgebras $C_{K}(\alpha)$ e $C_{K}(\beta)$, respectivamente. Se, além disso, $K$ é $D$-invariante, então pelo Lema 2.3.5, temos $K=K_{\alpha} \oplus K_{\beta}$.

Fixe $M=[L, L]$. Assim, para quaisquer $l \in M$ e $x, y \in L$, temos $[l, x, y]=[l, y, x]$.

Agora, dado um elemento $h \in L$, definimos os seguintes ideais de $L$ :

$$
C_{1}(h)=C_{M}(h), \quad \text { onde } C_{M}(h)=\{l \in M:[l, h]=0\}
$$

e

$$
C_{i+1}(h)=\left\{l \in M:[l, h] \in C_{i}(h)\right\}
$$

para $i$ maior do que 1 .

Nosso objetivo é provar que $M=[L, L]=C_{3 t-1}(z)$, para qualquer elemento homogêneo $z \in L$. Com isso obtemos que qualquer elemento homogêneo $z \in L$ é ad-nilpotente em $L$ de índice no máximo $3 t$, como desejamos. Note que precisamos provar apenas para elementos homogêneos de peso 1 , pois como $L$ é metabeliana, todo elemento homogêneo de peso maior ou igual a 2 é ad-nilpotente de índice no máximo 2. Por hipótese, qualquer elemento homogêneo $h_{\alpha} \in L_{\alpha}$ é ad-nilpotente de índice $t$ em $L_{\alpha}$, assim temos $M_{\alpha} \leq C_{t}\left(h_{\alpha}\right)$. Analogamente, temos $M_{\beta} \leq C_{t}\left(h_{\beta}\right)$. Assim, como $L$ é metabeliana, concluímos que $C_{t}\left(h_{\alpha}\right) \cap C_{t}\left(h_{\beta}\right)$ está contido em $C_{2 t-1}\left(h_{\alpha}+h_{\beta}\right)$ para elementos homogêneos arbitrários $h_{\alpha} \in L_{\alpha}$ e $h_{\beta} \in L_{\beta}$.

Seja $R$ o ideal gerado por $M_{\alpha}$. Embora, $R$ não necessariamente seja $D$-invariante, ainda temos $R=R_{\alpha}+R_{\beta}$. Na verdade, como $M=M_{\alpha}+M_{\beta}$, dado $x \in R$ podemos escrever $x=x_{1}+x_{2}$, onde $x_{1} \in M_{\alpha}$ e $x_{2} \in M_{\beta}$. Agora, levando em conta que $M_{\alpha}=R_{\alpha}$, concluímos que $x_{1} \in R$ e, consequentemente, $x_{2} \in R$. Assim, temos $R=R_{\alpha}+R_{\beta}$.

Note que $R_{\beta}$ está contido na interseção $C_{t}\left(h_{\alpha}\right) \cap C_{t}\left(h_{\beta}\right)$, para quaisquer elementos homogêneos $h_{\alpha} \in L_{\alpha}$ e $h_{\beta} \in L_{\beta}$. Agora, fixe um elemento homogêneo $z \in L$ de peso 1 , como a componente homogênea de peso 1 é $D$-invariante (pois os geradores são $D$ invariantes), existem elementos homogêneos $z_{\alpha} \in L_{\alpha}$ e $z_{\beta} \in L_{\beta}$ de peso um tais que $z=z_{\alpha}+z_{\beta}$. Diante disso, concluímos que $R_{\beta}$ está contido em $C_{2 t-1}(z)$. Em particular, isto mostra que $M_{\alpha}$ é igual a $R$ módulo $N=\bigcap_{i} C_{2 t-1}\left(z_{i}\right)$, onde $z_{i}$ percorre o conjunto 
de elementos homogêneos de peso 1 de $L$. Por um argumento "simétrico" concluímos o mesmo para $M_{\beta}$. Portanto, como $N$ é um ideal $D$-invariante, podemos passar para o quociente $L / N$ e assumir, sem perda de generalidade, que $M_{\alpha}$ e $M_{\beta}$ são ideais.

Seja $I$ o subgrupo aditivo de $L$ que consiste de todos os elementos $l \in L$ tais que $l^{\alpha}=-l$. Como a característica do corpo é ímpar, temos que $L=L_{\alpha} \oplus I$. Se $l \in I$ e $y \in M_{\alpha}$, então $[l, y]^{\alpha}=-[l, y]$ e assim $[l, y] \in I$. Por outro lado, como $M_{\alpha}$ é um ideal, $[l, y] \in M_{\alpha}$. Claramente temos $M_{\alpha} \cap I=0$ e assim $[l, y]=0$. Portanto, $I$ centraliza $M_{\alpha} \mathrm{e}$ temos $\left[M_{\alpha}, L\right]=\left[M_{\alpha}, L_{\alpha}\right]$ pois $L=L_{\alpha} \oplus I$. Isso nos permite concluir que para qualquer elemento homogêneo $z=z_{\alpha}+l$ onde $z_{\alpha} \in L_{\alpha}$ e $l \in I$ temos

$$
C_{1}\left(z_{\alpha}\right) \cap M_{\alpha} \leq C_{1}(z)
$$

Além disso, deduzimos que $C_{i}\left(z_{\alpha}\right) \cap M_{\alpha} \leq C_{i}(z)$. Como $M_{\alpha} \leq C_{t}\left(z_{\alpha}\right)$, temos $M_{\alpha} \leq$ $C_{t}(z)$. Similarmente, $M_{\beta} \leq C_{t}(z)$. Agora, como

$$
[L, L]=M=M_{\alpha}+M_{\beta},
$$

concluimos que $[L, L]=C_{t}(z)$. Portanto,

$$
[L, \underbrace{z, \ldots z}_{3 t}]=0
$$

para qualquer elemento homogêneo $z \in L$.

Agora demonstramos o principal resultado desta subseção.

Teorema 4.2.3. Assuma que um grupo diedral $D=\langle\alpha, \beta\rangle$ gerado por duas involuções $\alpha$ e $\beta$ aja sobre uma álgebra de Lie $L$ de tal modo que $C_{L}(\alpha \beta)=0$ e $|\alpha \beta|=n$. Seja $X \subset L$ um subconjunto D-invariante com d elementos tal que $L=\langle X\rangle$ e suponha que exista um inteiro positivo t para o qual todo elemento homogêneo contido em $C_{L}(\alpha)$ ou $C_{L}(\beta)$ é ad-nilpotente de indice no máximo t em $C_{L}(\alpha)$ ou $C_{L}(\beta)$, respectivamente. Então $L$ é nilpotente de classe $(d, n, t)$-limitada.

Demonstração. Pelo Teorema de Kreknin 1.8 .9 a álgebra de Lie $L$ é solúvel com comprimento derivado no máximo $2^{n-1}-1$. Assim, podemos usar indução sobre o comprimento derivado de $L$. Denote por $Y$ o conjunto de todos os elementos homogêneos de $L$. Claramente todos os termos da série central inferior e da série derivada de $L$ são gerados por elementos de $Y$.

Pelo Lema 4.2.2, se $L$ é metabeliana e gerada por no máximo $d$ elementos, então $L$ é nilpotente de classe $(d, t)$-limitada. Assim, podemos assumir que o comprimento derivado 
de $L$ é no mínimo três, digamos $f$. Seja $K=L^{(f-2)}$ e $M=[K, K]$. Note que $M$ é o último termo não trivial da série derivada de $L$ e que $K$ é um ideal $D$-invariante metabeliano de $L$. Por indução, $L / M$ é nilpotente de classe $(d, n, t)$-limitada. Em particular, isso implica que a dimensão de $L / M$ é finita e $(d, n, t)$-limitada. Diante disso, concluímos que existe um número $(d, n, t)$-limitado de elementos $x_{1}, \ldots, x_{u} \in Y \cap K$ tal que $K=\left\langle x_{1}, \ldots, x_{u}, M\right\rangle$ e pelo Lema 4.2.2, a subalgebra $\left\langle M, x_{i}\right\rangle$ é nilpotente de classe no máximo $m=3 t-1$, para cada $i=1, \ldots, u$.

Fixe $s=u(m-1)+1$ e considere o ideal

$$
S=[M, \underbrace{K, \ldots, K}_{s}] .
$$

Assim, $S$ é gerado por comutadores da forma $\left[l, y_{1}, \ldots, y_{s}\right]$ onde $l \in M$ e $y_{1}, \ldots, y_{s}$ são elementos, não necessariamente distintos, de $\left\{x_{1}, \ldots, x_{u}\right\}$. Como o número $s$ foi escolhido grande o suficiente, temos que existem $m$ índices $i_{1}, \ldots, i_{m}$ tais que temos $y_{i_{1}}=\cdots=y_{i_{m}}$. Como $M=[K, K]$, temos $[l, x, y]=[l, y, x]$, para todos $l \in M$ e $x, y \in K$. Portanto podemos assumir, sem perda de generalidade, que $y_{1}=\cdots=y_{m}$. Como $y_{1}$ é ad-nilpotente de índice no máximo $m$ em $\left\langle M, y_{1}\right\rangle$, temos $\left[l, y_{1}, \ldots, y_{s}\right]=0$. Assim, $S=0$, que significa que $M$ está contido no $s$-ésimo termo da série central inferior de $K$. Assim, o fato de que $M=[K, K]$ implica que $K$ é nilpotente de classe $(d, n, t)$-limitada. Portanto, $K$ e $L /[K, K]$ são nilpotentes de classe $(d, n, t)$-limitada. Agora, usando a versão do Teorema de P. Hall para álgebras de Lie 1.8.8, deduzimos que $L$ também é nilpotente de classe $(d, n, t)$-limitada.

\section{p-grupos admitindo um grupo diedral de automorfismos}

Ao longo desta seção admita que $D=\langle\alpha, \beta\rangle$ seja um grupo diedral gerado por duas involuções $\alpha$ e $\beta$. Além disso, suponha que $D$ aja sobre um $p$-grupo $G$ de tal maneira que $C_{G}(\alpha \beta)=1$.

Teorema 4.2.4. Seja p um primo e G um p-grupo potente. Então para todo número positivo e temos

$$
C_{G^{e}}(\alpha)=\left(C_{G}(\alpha)\right)^{e} \text { e } C_{G^{e}}(\beta)=\left(C_{G}(\beta)\right)^{e} .
$$

Consequentemente, se ambos $C_{G}(\alpha)$ e $C_{G}(\beta)$ são extensões de um grupo nilpotente de classe c por um grupo de expoente e, então $G$ é uma extensão de um grupo nilpotente de classe c-limitada por um grupo de expoente e. 
Demonstração. Claramente é suficiente provar que $C_{G^{e}}(\alpha) \leq\left(C_{G}(\alpha)\right)^{e}$ pois a outra inclusão é obvia. Sem perda de generalidade assumiremos que e é uma potência de $p$. Se $G$ possui expoente $p$, então não há nada para provar. Assim supomos que $G$ possua expoente $p^{k}$ onde $k \geq 2$. Temos que $e=p^{k-i}$ onde $i \in\{1, \ldots, k-1\}$ (o caso $i=0$ é trivial). Agora argumentamos por indução $i$. Se $i=1$, temos que $G^{e}=G^{p^{k-1}}$ e pelo Lema 1.2.4 a aplicação $\vartheta: G / G^{p} \longrightarrow G^{e}$ tal que $x \longmapsto x^{e}$ é um homomorfismo. O Lema 2.3.4 mostra que todo elemento de $C_{G^{e}}(\alpha)$ é uma imagem de algum elemento de $C_{G / G^{p}}(\alpha)$ e que todo elemento de $C_{G / G^{p}}(\alpha)$ é uma imagem de algum elemento de $C_{G}(\alpha)$. Assim, para todo $x \in C_{G^{e}}(\alpha)$, existe $y \in C_{G}(\alpha)$ tal que $y^{e}=x$. Agora suponha $i>1$. A aplicação $x \longmapsto x^{e}$ é um homomorfismo de $G / G^{p}$ em $G^{e} / G^{p^{k-(i-1)}}$, pelo Lema 1.2.4. Por um argumento similar, para todo elemento $x \in C_{G^{e}}(\alpha)$ existe $y \in C_{G}(\alpha)$ tal que $y^{e}=x z$ onde $z \in C_{G^{p^{k-(i-1)}}}(\alpha)$. Portanto, como

$$
z=h^{p^{k-(i-1)}}=\left(h^{p}\right)^{p^{k-i}},
$$

onde $h \in C_{G}(\alpha)$, temos

$$
C_{G^{e}}(\alpha)=\left(C_{G}(\alpha)\right)^{e}
$$

e similarmente

$$
C_{G^{e}}(\beta)=\left(C_{G}(\beta)\right)^{e}
$$

Consequentemente, se ambos $\left(C_{G}(\alpha)\right)^{e}$ e $\left(C_{G}(\beta)\right)^{e}$ são nilpotentes de classe no máximo $c$, então pelo Teorema 2.2.2, provado por Shumyatsky, o subgrupo normal $G^{e}$ é nilpotente de classe $c$-limitada como queríamos demonstrar.

Na demonstração do próximo teorema utilizaremos a solução do Problema Restrito de Burnside provada por Zelmanov em [42, 43]. Tal solução nos diz que a quantidade de grupos finitos $d$ gerados com expoente $e$ é $(d, e)$-limitada.

Teorema 4.2.5. Seja p um número primo. Suponha que $G$ seja um p-grupo finito $d$ gerado, no qual o conjunto gerador é D-invariante. Admita que ambos $C_{G}(\alpha)$ e $C_{G}(\beta)$ sejam extensões de um grupo nilpotente de classe no máximo c por um grupo de expoente e. Então $G$ possui um subgrupo característico nilpotente de classe c-limitada e indice $(c, d, e, p)$-limitado.

Demonstração. Primeiro, consideramos $p=2$ e, provamos que $G$ possui um subgrupo potente de índice $(c, d, e, p)$-limitado. Neste caso podemos assumir que $D$ é um grupo de Frobenius. Mais ainda, como $C_{G}(\alpha)$ e $C_{G}(\beta)$ são extensões de um grupo nilpotente de classe no máximo $c$ por um grupo de expoente $e$ temos que $C_{G}(\alpha)$ e $C_{G}(\beta)$ satisfazem uma lei positiva de grau $(c, e)$-limitado. Na verdade, $C_{G}(\alpha)$ e $C_{G}(\beta)$ satisfazem a lei positiva 
$M_{c}\left(x^{e}, y^{e}\right)$ onde $M_{c}(x, y)$ é a lei de Malcev. Portanto usando o Teorema 4.0.1 e o resultado de Burns, Macedońska e Medvedev 1.3.1 concluímos que $G$ possui um subgrupo $N$ cuja classe de nilpotência e o expoente de $G / N$ dependem apenas de $c$ e $e$. Agora, usando a solução do Problema Restrito de Burnside, concluímos que o índice de $N$ em $P$ é $(c, d, e)$ limitado. Uma consequência disso é que o posto de $G$ também é $(c, d, e)$-limitado. Agora, concluímos a demonstração, para $p=2$, usando o Teorema 1.2.6.

Agora, consideramos $p>2$ e provamos que $G$ também possui um subgrupo potente de índice $(c, d, e, p)$-limitado. Sejam $X$ um conjunto de geradores de $G$ e $L=L_{p}(G)$. A álgebra $L$ é gerada pela imagem $\bar{X}$ de $X$. Claramente um elemento de $L$ é homogêneo com respeito a $\bar{X}$ se, e somente se, ele é homogêneo com respeito a $L_{1}$. O grupo $D=\langle\alpha, \beta\rangle$ age naturalmente sobre todo quociente $L_{i}=D_{i} / D_{i+1}$ e a ação se estende para $D L(G)$ e $L$. Assim, podemos ver $D$ como um grupo de automorfismos de L. Pelo Lema 2.3.4, temos $C_{L}(\alpha \beta)=0$,

$$
C_{L}(\alpha)=\bigoplus C_{D_{i}}(\alpha) D_{i+1} / D_{i+1}
$$

e

$$
C_{L}(\beta)=\bigoplus C_{D_{i}}(\beta) D_{i+1} / D_{i+1}
$$

Assim, como ambos $C_{G}(\alpha)$ e $C_{G}(\beta)$ são extensões de um grupo nilpotente de classe no máximo $c$ por um grupo de expoente $e$, deduzimos do Corolário 1.9 .2 que se $x$ é um elemento homogêneo de $C_{L}(\alpha)$ ou $C_{L}(\beta)$, então $x$ é ad-nilpotente de índice no máximo $(c+1) e$ em $C_{L}(\alpha)$ ou $C_{L}(\beta)$, respectivamente.

Portanto, podemos usar o Teorema 4.2 .3 e concluir que $L$ é nilpotente de classe $(c, d, e)$ limitada. Sendo assim, usando o Teorema 1.9.3, concluímos que $G$ possui um subgrupo potente característico $R$ de índice $(c, d, e, p)$-limitado.

Agora, sabemos que para qualquer primo $p$, o grupo $G$ possui um subgrupo potente $R$ de índice $(c, d, e, p)$-limitado. Levando em conta que $C_{R}(\alpha)$ e $C_{R}(\beta)$ são extensões de um grupo nilpotente de classe no máximo $c$ por um grupo de expoente $e$, podemos usar o Teorema 4.2.4 e o Teorema 2.2.2 para concluirmos que $R^{e}$ é nilpotente de classe $c$-limitada. Claramente $R^{e}$ é característico e possui índice $(c, d, e, p)$-limitado, o que completa a demonstração.

\section{Prova do teorema 4.2.1}

Agora já temos todos os resultados auxiliares necessários para provar o Teorema 4.2.1. Primeiro provamos o caso onde $G$ é nilpotente. 
Teorema 4.2.6. Suponha que um grupo Diedral $D=\langle\alpha, \beta\rangle$ aja sobre um grupo nilpotente finito $G$ de tal modo que $C_{G}(\alpha \beta)=1$ e ambos $C_{G}(\alpha)$ e $C_{G}(\beta)$ satisfazem uma lei positiva de grau $k$. Então $G$ satisfaz uma lei positiva de grau limitado apenas em termos de $k e$ $|D|$.

Demonstração. De acordo com o resultado de Burns, Macedońska e Medvedev 1.3.1, existem inteiros $c=c(k)$ e $e=e(k)$, dependendo somente de $k$, tais que ambos $C_{G}(\alpha)$ e $C_{G}(\beta)$ são extensões de um grupo nilpotente de classe no máximo $c$ por um grupo de expoente dividindo $e$. Primeiro suponha que $G$ seja um $p$-grupo para algum primo $p$. Se $p=2$, então podemos assumir que $|\alpha \beta|$ é ímpar, pelo Lema 1.4.3 e assim $D$ é um grupo de Frobenius. Neste caso, o resultado segue do Teorema 4.0.1 provado por Shumyatsky. Assim, podemos assumir que $p$ é ímpar. Se $p$ não divide $e$, então $C_{G}(\alpha)$ e $C_{G}(\beta)$ são necessariamente nilpotentes de classe no máximo $c$, neste caso, o resultado segue pelo Teorema 2.2.2, pois $G$ deve ser nilpotente de classe $c$-limitada. Portanto podemos assumir que $p$ é um divisor de e assim $p$ é $k$-limitado. Desejamos mostrar que $G$ satisfaz uma lei positiva de duas variáveis com grau limitado. Para isto é suficiente provar o teorema para todo subgrupo 2-gerado de $G$. Como todo subgrupo 2-gerado de $G$ está contido em um subgrupo $D$-invariante com no máximo $2|D|$ geradores, sem perda de generalidade podemos assumir que $G$ pode ser gerado por um conjunto $D$-invariante $X$ de $d \leq 2|D|$ elementos.

Assim, pelo Teorema 4.2.5 o grupo $G$ é uma extensão de um grupo nilpotente de classe $c$-limitada, digamos $c^{*}$, por um grupo de expoente $(k,|D|)$-limitado $e^{*}$. Se $M_{r}(x, y)$ denota a Lei de Malcev de grau $2^{r}$, concluímos que $G$ satisfaz a lei positiva $M_{c^{*}}\left(x^{e^{*}}, y^{e^{*}}\right)$ de grau $(k,|D|)$-limitado. Como tal lei não depende de $p$, concluímos que o resultado vale para todos os grupos finitos nilpotentes.

Como anteriormente, denotamos por $F(G)$ o subgrupo de Fitting e por $h(G)$ a altura de Fitting de um grupo $G$. Além disso, como de costume, aqui denotaremos por $\pi(G)$ o conjunto de primos divisores da ordem de $G$.

Prova do teorema 4.2.1: Como na prova do Teorema 4.2.6, existem inteiros $c=c(k)$ e $e=e(k)$ dependendo somente de $k$ tais que $C_{G}(\alpha)$ e $C_{G}(\beta)$ são extensões de um grupo nilpotente de classe no máximo $c$ por um grupo de expoente $e$. Além disso, como na prova do Teorema 4.2.6 podemos assumir que $G$ pode ser gerado por $d \leq 2|D|$ elementos. Como $\alpha \beta$ age sobre $G$ livre de pontos fixos, concluímos que $G$ é solúvel e sua altura de Fitting $h(G)$ é limitado por uma função que depende apenas de $|\alpha \beta|$ e assim podemos usar indução sobre o comprimento de Fitting de $G$. Se $h(G)=1$, o resultado é imediato pelo Teorema 
4.2.6. Agora, assuma que $h(G)=2$, ou seja, que $G$ é metanilpotente. Fixe $M=F(G)$ e suponha que $\pi(G)$ contenha um primo $p$ que não divide $e$. Seja $P$ o $p$-subgrupo de Sylow $D$-invariante cuja existência é garantida pelo Lema 2.3.3. O subgrupo $M P$ é $D$ invariante. Como $p$ não divide $e$, temos que $C_{P}(\alpha) \leq F\left(C_{G}(\alpha)\right)$ e $C_{P}(\beta) \leq F\left(C_{G}(\beta)\right)$. Portanto, $C_{M P}(\alpha)$ e $C_{M P}(\beta)$ são nilpotentes e assim o Teorema 2.2.2 nos diz que $M P$ é nilpotente. Como $M P$ é normal, concluímos que $P \leq M$. Isto mostra que $\pi(G / M)$ consiste de divisores de e assim qualquer $p \in \pi(G / M)$ é limitado.

Primeiro suponha que $\pi(G / M)$ possui apenas um primo $p$. Seja $P$ o $p$-subgrupo de Sylow $D$-invariante de $G$. Temos que $G=M P$. Como $G / O_{\pi^{\prime}}(G)$ é isomorfo a $P$ e pode ser gerado por no máximo $d$-elementos, deduzimos que $P$ pode ser gerado por no máximo $d$ elementos. Como $C_{P}(\alpha) \leq C_{G}(\alpha)$ e $C_{P}(\beta) \leq C_{G}(\beta)$, concluímos que $C_{P}(\alpha)$ e $C_{P}(\beta)$ são extensões de um grupo nilpotente de classe no máximo $c$ por um grupo de expoente dividindo $e$. Então, pelo Teorema 4.2.5, $P$ contém um subgrupo característico potente $R$ de índice $(c,|D|)$-limitado. Como $p$ é $k$-limitado, $T=M R^{e}$ possui índice $(k,|D|)$-limitado em $G$. Além disso, pelo Teorema 4.2.4 $C_{R^{e}}(\alpha)=\left(C_{R}(\alpha)\right)^{e} \leq F\left(C_{G}(\alpha)\right)$ e $C_{R^{e}}(\beta)=\left(C_{R}(\beta)\right)^{e} \leq F\left(C_{G}(\beta)\right)$. Disso segue que $C_{T}(\alpha)$ e $C_{T}(\beta)$ são nilpotentes. Assim, usando o Teorema 2.2.2, concluímos que $T$ é nilpotente e consequentemente $T=M$.

Mostramos que no caso em que $\pi(G / M)$ possui apenas um primo, o índice de $M$ em $G$ é $(k,|D|)$-limitado. Por outro lado, sabemos que $|\pi(G / M)|$ é $(k,|D|)$-limitado. Considere que $\pi(G / M)=\left\{p_{1}, \ldots, p_{s}\right\}$ e, para cada $i=1, \ldots, s$, escolha um $p_{i}$-subgrupo de Sylow $P_{i}$ $D$-invariante de $G$. De acordo com os argumentos acima, $M$ possui índice $(k,|D|)$-limitado em todo subgrupo $M P_{i}$. Portanto, levando em conta que o número de tais subgrupos é $(k,|D|)$-limitado, concluímos que o índice de $M$ em $G$ é $(k,|D|)$-limitado. De acordo com o Teorema 4.2.6, $M$ satisfaz uma lei positiva de grau $(k,|D|)$-limitado e com isso concluímos que $G$ também. Isto prova o teorema no caso particular em que $h(G)=2$.

Agora assuma $h(G) \geq 3$ e fixe novamente $M=F(G)$. Por hipótese de indução, assumimos que o quociente $G / M$ satisfaz uma lei positiva de grau que é limitado somente em termos de $k$ e $|D|$. De acordo com o resultado de Burns, Macedońska e Medvedev 1.3.1, $G$ possui um subgrupo normal $N$, contendo $M$, tal que $N / M$ é nilpotente de classe $(k,|D|)$-limitado e $G / N$ possui expoente $(k,|D|)$-limitado. Portanto, é suficiente mostrar que $N$ satisfaz uma lei positiva de grau $(k,|D|)$-limitado. No entanto isto é imediato do parágrafo anterior desde que $N$ é metanilpotente. 


\section{Capítulo}

\section{Considerações finais}

Se um grupo $A$ age como grupo de automorfismos sobre um grupo finito $G$, de modo que $C_{G}(A)=1$, então, frequentemente, podemos dizer alguma coisa sobre a estrutura de $G$ dadas propriedades de $A$. Recentemente, Khukhro, Makarenko, Shumyatsky, entre outros autores, tem considerado o caso em que $A$ contém um subgrupo normal nilpotente $B$ tal que $C_{G}(B)=1$ e, tem perguntado: Propriedades de $G$ estão relacionadas com as respectivas propriedades do subgrupo dos pontos fixos dos elementos fora do subgrupo $B$ ?

Conforme resultados citados no decorrer deste trabalho, respostas afirmativas foram obtidas, por exemplo, para os casos em que $A$ é um grupo de Frobenius, um grupo diedral e um grupo Frobenius-like. As propriedades consideradas foram, nilpotência, expoente, lei positiva, altura de Fitting, entre outras.

No presente trabalho, estendemos alguns resultados, conhecidos anteriormente apenas para grupos de Frobenius, grupos diedral e grupos Frobenius-like, para uma nova "família" de grupos metacíclicos. Além disso, provamos novos resultados para grupos admitindo um grupo diedral de automorfismos.

Encerramos este trabalho apresentando algumas questões. Elas estão relacionados com os resultados apresentados ao longo de todo o trabalho e permanecem sem repostas até o momento.

Questão 1: Seja $M=F H$ um grupo finito o qual é um produto de dois subgrupos cíclicos $F$ e $H$, onde $F$ é um subgrupo normal e todos os elementos de $M \backslash F$ possuem ordem prima $p$. Suponha que $M$ aja sobre um grupo finito $G$ de tal maneira que $C_{G}(F)=$ 1.

(a) Admita que $C_{G}(x)$ seja nilpotente, para todos $x \in M \backslash F$. A classe de nilpotência de $G$ é limitada em termos de p e da classe de nilpotência dos subgrupos $C_{G}(x)$ ? 
(b) Se $C_{G}(x)$ satisfaz uma lei positiva de grau $k$, para todos $x \in M \backslash F$, será que $G$ satisfaz uma lei positiva de grau $(k, p)$-limitado?

Lembre-se que pelo Corolário 3.0.10, no Item (a), o grupo $G$ deve ser nilpotente.

A próxima questão surge naturalmente na tentativa de estender os resultados, válidos para grupos com um grupo diedral de automorfismos, para grupos com um p-grupo de classe maximal de automorfismos. No entanto, devemos lembrar que o Exemplo 3.0.11 nos diz que, de um modo geral, o Fitting do centralizador de um elemento não pertence ao Fitting do grupo. Em tal exemplo, a altura de Fitting do centralizador é 2, assim resta estudar o caso em que o centralizador é necessariamente nilpotente.

Questão 2: Seja $A=N B$ um grupo finito o qual é o produto de um subgrupo normal nilpotente $N$ e um subgrupo $B$ de ordem prima $p$ tal que $\left|C_{A}(B)\right|=p^{2}$. Suponha que $A$ aja sobre um grupo finito $G$ de tal maneira que $C_{G}(N)=1$.

(a) Se $C_{G}(B)$ é nilpotente, será que o subgrupo $C_{G}(B)$ pertence ao subgrupo de Fitting de $G$ ?

(b) Se $x^{p}=1,\left|C_{A}(x)\right|=p^{2}$ e $C_{G}(x)$ é nilpotente, para todos $x \in A \backslash N$, será que $G$ é nilpotente?

Respondemos de forma afirmativa a questão 2, para o caso em que $A$ é um $p$-grupo. Neste caso, a hipótese $\left|C_{A}(B)\right|=p^{2}$ implica que $A$ é um $p$-grupo de classe maximal e, as hipóteses $x^{p}=1$ e $\left|C_{A}(x)\right|=p^{2}$, do Item (b), significam que todos elementos fora do subgrupo $N$ são "uniformes" de ordem $p$. Para mais detalhes sobre $p$-grupos de classe maximal veja [14]. 


\section{Referências Bibliográficas}

[1] C. Acciarri, P. Shumyatsky, Double automorphisms of graded Lie algebras, J. Algebra 387 (2013), 1-10.

[2] V. V. Belyaev, B. Hartley, Centralizers of finite nilpotent subgroup in locally finite groups, Algebra Logika 35 (1996), 389-410; English transl., Algebra Logic 35 (1996), 217-228.

[3] R. G. Burns, O. Macedońska, Yu. Medvedev, Groups satisfying semigroup laws, and nilpotent-by-Burnside varieties, J. Algebra 195 (1997), 510-525.

[4] J. Caldeira, E. de Melo, P. Shumyatsky, On groups and Lie algebras admitting a Frobenius group of automorphisms, J. Pure and Appl. Algebra 216 (2012), 27302736 .

[5] G. Collins, P. Flavell, On fixed-point-free automorphisms, J. Algebra 423 (2015), 798-811.

[6] C. W. Curtis and I. Reiner, Representation theory of finite groups and associative algebras, Interscience, New York-London, 1962.

[7] E. C. Dade, Carter subgroups and Fitting heights of finite solvable groups, Illinois J. Math. 13 (1969), 449-514.

[8] E. de Melo, Positive laws in finite groups admitting a dihedral group of automorphisms, J. Group Theory 16 (2013), 767-778.

[9] E. de Melo, Fitting height of a finite group with a metabelian group of automorphisms Comm. Alg. To appear DOI:10.1080/00927872.2014.952737

[10] J. D. Dixon, M.P.F. du Sautoy, A. Mann, D. Segal, Analytic Pro-p Groups, in: London Math. Soc. Lecture Note Series, vol. 157, Cambridge Univ. Press, 1991. 
[11] G. Ercan, I. Guloglu, E. Ogut, Nilpotent length of a finite solvable group with a Frobenius group of automorphisms, Comm. Algebra. To appear.

[12] G. Ercan, I. Guloglu, Action of a Frobenius-Like group with fixed-point-free kernel, J. Group Theory 17 (2014), 863-873.

[13] G. Ercan, I. Guloglu, E. Khukhro, Rank and Order of a Finite Group admitting a Frobenius-Like Group of Automorphisms, Alg. Logic. 53 (2014), 258-265.

[14] G. A. Fernández Alcober, An inroduction to finite p-groups: Regular p-groups and groups of maximal class, Matemática Contemporânea, 20 (2001), 155-226.

[15] D. Gorenstein, Finite Groups, Harper and Row, New York, 1968.

[16] D. Gorenstein, Finite Simple Groups: An Introduction to their Classification, Plenum Press, New York, London, 1982.

[17] G. Higman, Groups and rings which have automorphisms without non-trivial fixed elements, J. London Math. Soc. (2) 32 (1957), 321-334.

[18] P. Hall, Some sufficient conditions for a group to be nilpotent, Illinois. J. Math 2 (1958), 787-801.

[19] E. I. Khukhro, P. Shumyatsky, Bounding the exponent of a finite group with automorphisms, J. Algebra 212 (1999) 363-374.

[20] E. I. Khukhro, Nilpotent length of a finite group admitting a Frobenius group of automorphisms with fixed-point-free kernel, Algebra and Logic. 49 (2011), 551-560.

[21] E. I. Khukhro, Fitting height of a finite group with a Frobenius group of automorphisms, J. Algebra 366 (2012), 1-11.

[22] E. I. Khukhro, Rank and order of a finite group admitting a Frobenius group of automorphisms, Algebra Logika. 52 (2013), 99-108; English transl. Algebra Logic. 52 (2013), 72-78.

[23] E. I. Khukhro, N. Y. Makarenko, Finite groups and Lie rings with a metacyclic Frobenius group of automorphisms J. Algebra. 386 (2013), 77-104.

[24] E. I. Khukhro, N. Y. Makarenko, Finite p-groups admitting a Frobenius group of automorphisms whose kernel is a cyclic p-group Proc. Amer. Math. (2014), To appear.

[25] E. I. Khukhro, N. Y. Makarenko, P. Shumyatsky, Frobenius groups of automorphisms and their fixed points, Forum Math. 26 (2014), 73-112. 
[26] E. I. Khukhro, p-Automorphisms of Finite p-groups, in: London Mathematical Society Lecture Note Series, vol.246, 1998.

[27] E. I. Khukhro, Nilpotent Groups and their Automorphisms, de Gruyter-Verlag, Berlin, 1993.

[28] E. I. Khukhro, P. Shumyatsky, Nilpotency of Finite Groups with Frobenius Groups of Automorphisms, Monatsh. Math. (2010), DOI: 10.1007/s00605-010-0198-8.

[29] V. A. Kreknin, The solubility of Lie algebras with regular automorphisms of finite period, Dokl. Akad. Nauk SSSR 150 (1963), 467-469 (Russian), Math. USSR Doklady 4 (1963), 683-685 (English).

[30] M. Lazard, Sur les groupes nilpotent et les anneaux de Lie, Ann. Sci. É. Norm. Supré. 71 (1954) 101-190.

[31] A. Lima, P. Shumyatsky, On groups satisfying a positive laws in fixed points, J. Algebra 322 (2009), 245-253.

[32] A. I. Malcev, Nilpotent semigroups, Uchen. Zap. Ivanovsk. Ped. Inst. 4 (1953), 107111.

[33] N. Yu. Makarenko, P. Shumyatsky, Frobenius groups as groups of automorphisms, Proc. Amer. Math. Soc. 138 (2010) 3425-3436.

[34] A. Yu. Olshansky, A. Storozhev, A group variety defined by a semigroup law, J. Austral Math. Soc. Ser. A 60 (1996), 255-259.

[35] D. J.S. Robinson, A course in the theory of groups, 2nd edition, Springer-Verlag, New York, 1996.

[36] P. Shumyatsky, Positive laws in fixed points, Trans. Amer. Math. Soc. 356(5) (2004), 2081-2091.

[37] P. Shumyatsky, Positive laws in derived subgroups of fixed points, Q. J. Math. 60(1) (2009), 121-132.

[38] P. Shumyatsky, Positive laws in fixed points of automorphisms of finite groups, J. Pure and Appl. Algebra 215 (2011), 2559-2566.

[39] P. Shumyatsky, The dihedral group as a group of automorphisms, J. Algebra. 366 (2013), 1-12. 
[40] J. G. Thompson, Finite groups with fixed-point-free automorphisms of prime order, Proc. Natl. Acad. Sci. USA 45 (1959), 578-581.

[41] A. Turull, Fitting Height of Groups and of Fixed points, J. Algebra 86 (1984), 555566.

[42] E. Zelmanov, The solution of the restricted Burnside problem for groups of odd exponent, Math. USSR Izv. 36 (1991), 41-60.

[43] E. Zelmanov, The solution of the restricted Burnside problem for 2-groups, Math. Sb. 182 (1991) 568-592.

[44] Unsolved Problems in Group Theory. The Kourovka Notebook, no. 17, Institute of Mathematics, Novosibirsk, 2010. 